\title{
Grafting Embryonic Raphe Neurons Reestablishes Serotonergic Regulation of Sympathetic Activity to Improve Cardiovascular Function after Spinal Cord Injury
}

\author{
(DShaoping Hou, Tatiana M. Saltos, $\odot$ Eugene Mironets, Cameron T. Trueblood, Theresa M. Connors, and ๑Veronica J. Tom \\ Marion Murray Spinal Cord Research Center, Department of Neurobiology \& Anatomy, Drexel University College of Medicine, Philadelphia, Pennsylvania \\ 19129
}

Cardiovascular dysfunction often occurs after high-level spinal cord injury. Disrupting supraspinal vasomotor pathways affects basal hemodynamics and contributes to the development of autonomic dysreflexia (AD). Transplantation of early-stage neurons to the injured cord may reconstruct the descending projections to enhance cardiovascular performance. To determine the specific role of reestablishing serotonergic regulation of hemodynamics, we implanted serotonergic $\left(5-\mathrm{HT}^{+}\right)$neuron-enriched embryonic raphe nucleus-derived neural stem cells/progenitors (RN-NSCs) into a complete spinal cord transection lesion site in adult female rats. Grafting embryonic spinal cord-derived NSCs or injury alone served as 2 controls. Ten weeks after injury/grafting, histological analysis revealed well-survived grafts and partial integration with host tissues in the lesion site. Numerous graft-derived serotonergic axons topographically projected to the caudal autonomic regions. Neuronal tracing showed that host supraspinal vasomotor pathways regenerated into the graft, and 5- $\mathrm{HT}^{+}$ neurons within graft and host brainstem neurons were transsynaptically labeled by injecting pseudorabies virus (PRV-614) into the kidney, indicating reconnected serotonergic circuits regulating autonomic activity. Using an implanted telemeter to record cardiovascular parameters, grafting RN-NSCs restored resting mean arterial pressure to normal levels and remarkably alleviated naturally occurring and colorectal distension-induced AD. Subsequent pharmacological blockade of 5- $\mathrm{HT}_{2 \mathrm{~A}}$ receptors with ketanserin in RN-NSCgrafted rats reduced resting mean arterial pressure and increased heart rate in all but 2 controls. Furthermore, spinal cord retransection below RN-NSC grafts partially eliminated the recovery in AD. Collectively, these data indicate that RN-NSCs grafted into a spinal cord injury site relay supraspinal control of serotonergic regulation for sympathetic activity to improve cardiovascular function.

Key words: cell transplantation; hemodynamics; spinal cord transection; transsynaptic tracing

Significance Statement

Disruption of supraspinal vasomotor pathways results in cardiovascular dysfunction following high-level spinal cord injury. To reestablish the descending regulation of autonomic function, we transplanted serotonergic neuron enriched embryonic raphe nucleus-derived neural stem cells/progenitors into the lesion site of completely transected rat spinal cord. Consequently, grafted raphe nucleus-derived neural stem cells/progenitors acted as a neuronal relay to reconnect supraspinal center and spinal sympathetic neurons below the injury. The reconstituted serotonergic regulation of sympathetic activity led to the improvement of hemodynamic parameters and mitigated autonomic dysreflexia. Based on morphological and physiological results, this study validates the effectiveness of transplanting early-stage serotonergic neurons into the spinal cord for cardiovascular functional recovery after spinal cord injury.

\section{Introduction}

Cardiovascular complications are one of the leading causes of mortality and morbidity in those with a spinal cord injury
(SCI). After a severe, high-level SCI, patients may suffer from myriad manifestations of cardiovascular dysfunctions, including altered resting hemodynamics, orthostatic hypotension, and 
life-threatening autonomic dysreflexia $(\mathrm{AD})$. Episodic $\mathrm{AD}$ is clinically defined as acute hypertension induced by a massive, sympathetic discharge that is often accompanied by baroreceptormediated bradycardia (Karlsson, 1999; Krassioukov et al., 2003). $\mathrm{AD}$ events are commonly triggered by noxious visceral or somatic sensory stimulation below the injury (Teasell et al., 2000; Mathias, 2006; Eldahan and Rabchevsky, 2018). While multiple mechanisms have been associated with the development of cardiovascular dysfunction following SCI, including sprouting of pelvic afferents (Krenz and Weaver, 1998; Cameron et al., 2006), propriospinal plasticity (Rabchevsky, 2006; Hou et al., 2008), and increased sensitivity of adrenergic receptors in peripheral blood vessels (Krassioukov et al., 2002), a primary cause is the disruption of descending vasomotor pathways.

The majority of supraspinal regions have been characterized to provide input to sympathetic preganglionic neurons (SPNs) and regulate their activity in the thoracolumbar spinal cord, for example, originating from the rostral ventrolateral medulla (RVLM), rostral ventromedial medulla, caudal raphe nuclei (RN), A5 region, and paraventricular nucleus of the hypothalamus (Jansen et al., 1995). These descending projections release a variety of neurotransmitters and neuropeptides acting upon SPNs to regulate blood pressure and heart rate (Furlan et al., 2003; LlewellynSmith, 2009). Among them, the caudal RN, which are composed of the raphe magnus, raphe pallidus, and raphe obscurus, extend the main serotonergic innervation to SPNs in the spinal cord (Ahn et al., 2018). The pathways release serotonin (5-HT) that plays an essential role in regulating sympathetic outflow (Calaresu and Yardley, 1988; Coote, 1990). Previous studies reported that descending serotonergic axons can independently produce a pressor response (Chalmers et al., 1988). It was also demonstrated that the loss of serotonergic innervation and regulation following SCI is one important reason for disordered hemodynamics (Cormier et al., 2010). Accordingly, we postulated that reestablishing serotonergic regulation of SPN activity will improve cardiovascular function following SCI.

In recent decades, many studies were focused on promoting axon regeneration to restore function following SCI, but physiological improvements are subtle or modest, even when both intrinsic regenerative capacity and extrinsic inhibitory environments were extensively manipulated (Tuszynski and Steward, 2012; Wu et al., 2017). By contrast, transplantation of neural stem cells into the lesion of an injured spinal cord remains a promising therapeutic strategy to repair compromised function. Unlike mature central neurons with a poor capacity to regenerate, developing ones often express high levels of growth-related proteins that permit extensive axon elongation. Previous investigations showed that, when multipotent progenitor cells derived from either embryonic spinal cord or stem cells were implanted into the rat injured spinal cord, they extended long projections and eventually differentiated into mature neurons (Reier et al., 1992; Cummings et al., 2005; Lu et al., 2012; Kadoya et al., 2016). Newly differentiated neurons can then form relay circuits to compensate for disrupted supraspinal pathways (Bonner et al., 2011). We recently transplanted embryonic brainstem-derived neural stem cells/progenitors (BS-NSCs) to the injured spinal cord and found

Dr. Lynn Enquist (Princeton University) as part of Virus (enter funding (P40 RR018604) awarded to Dr. Enquist. These two reagents were generously provided by Dr. Michael A. Lane (Drexel University).

The authors declare no competing financial interests.

Correspondence should be addressed to Veronica J. Tom at vjt25@drexel.edu or Shaoping Hou at sh698@drexel.edu.

https://doi.org/10.1523/JNEUROSCI.1654-19.2019

Copyright $\odot 2020$ the authors recovered resting blood pressure and alleviated artificially induced AD (Hou et al., 2013b). Histologically, some grafted $5-\mathrm{HT}^{+}$neurons survived and projected to the caudal spinal cord, correlating with the improved sympathetic tone and diminished $\mathrm{AD}$.

In the present study, we sought to more specifically determine the role of reestablishing serotonergic innervation to abrogate cardiovascular dysfunction following SCI. By transplanting neural stem cells/progenitors derived from the embryonic raphe (RN-NSCs) into a completely transected spinal cord, we aimed to reconstitute serotonergic modulation of sympathetic activity for cardiovascular functional improvement after SCI.

\section{Materials and Methods}

Animals

Adult female Fischer 344 (F344) rats (150-200 g, 2 months old) were used in the experiments. Institutional Animal Care and Use Committee and Society for Neuroscience guidelines on animal care were strictly followed to minimize the number of animals used and any potential suffering. For all surgeries, including SCI and cell transplantation, neuronal tract tracer injection, implantation of telemetric transmitters, and spinal cord retransection, animals were anesthetized with inhalation of $4 \%$ isoflurane. Anesthesia was maintained using $2 \%$ isoflurane during the surgery.

Animals were divided into four groups: (1) transplanted with embryonic RN-NSCs; (2) transplanted with embryonic spinal cord-derived neural stem cells/progenitors (SC-NSCs) containing very few to no $5-\mathrm{HT}^{+}$neurons as a cellular control; (3) injury only without graft as another control; and (4) a naive, uninjured control. Some animals grafted with RN-NSCs underwent anterograde neural tracing, transsynaptic tracing, and spinal cord retransection 10 weeks after transplantation. To exclude the cases with incomplete SCI in all groups, we performed histological examination in the spinal cord sections. Animals with an incomplete lesion, which was identified by immunolabeling for GFAP in the lesion site of injury-only cords or $\mathrm{GFAP}^{+}$cells within $\mathrm{GFP}^{-}$ areas in the lesion of grafted cords, were ruled out $(n=6)$. In a few rats, graft integration in the lesion site was poor without axon extension into the host cord. They were also excluded regardless of their cardiovascular performance (RN-NSC graft, $n=3$; SC-NSC graft, $n=2$ ). Thus, a final number of 42 rats was used for histological and cardiovascular analysis (RN-NSC graft, $n=25,8$ for cardiovascular and histological analysis, 5 for anterograde tracing, 8 for transsynaptic tracing, 4 for spinal cord retransection; SC-NSC graft, $n=9,6$ for cardiovascular and 3 of them and other 3 for histological analysis; injury controls, $n=8$ for cardiovascular analysis, $n=6$ for histological analysis; naive rats, $n=6$ used to assess normal cardiovascular parameters).

\section{Cell preparation}

Previous studies demonstrated the role of central 5-HT in regulating hemodynamics and AD (Chalmers et al., 1988). Recently, we reported that grafting embryonic brainstem neurons reconstructs neuronal circuits and improves cardiovascular function (Hou et al., 2013b). To identify the specific effect of reestablished serotonergic regulation on the functional recovery, in the present study, we transplanted embryonic day 14 (E14) RN-NSCs enriched with serotonergic neurons into the injured spinal cord, whereas E14 SC-NSCs lacking adequate serotonergic neurons served as a cellular control. E14 brainstem area containing the rostral and caudal RN (Fig. 1A-C) or spinal cord from transgenic F344 transgenic rats ubiquitously expressing enhanced GFP under the ubiquitin $\mathrm{C}$ promoter provided donor tissue (Rat Resource and Research Center, University of Missouri, Columbia, Missouri). At this stage, embryonic grafts are composed of a mixture of NSCs, neuronal or glial restricted precursors (Kalyani and Rao, 1998). To maximally preserve extracellular matrixes to better support graft survival, GFP-expressing E14 RN or SC was freshly dissected and mechanically dissociated to small chucks instead of single-cell solution for implantation based on our recent findings (Hou et al., 2018). The tissue blocks were then resuspended in a fibrin matrix ( $25 \mathrm{mg} / \mathrm{ml}$ fibrinogen, $25 \mathrm{U} / \mathrm{ml}$ thrombin) containing 

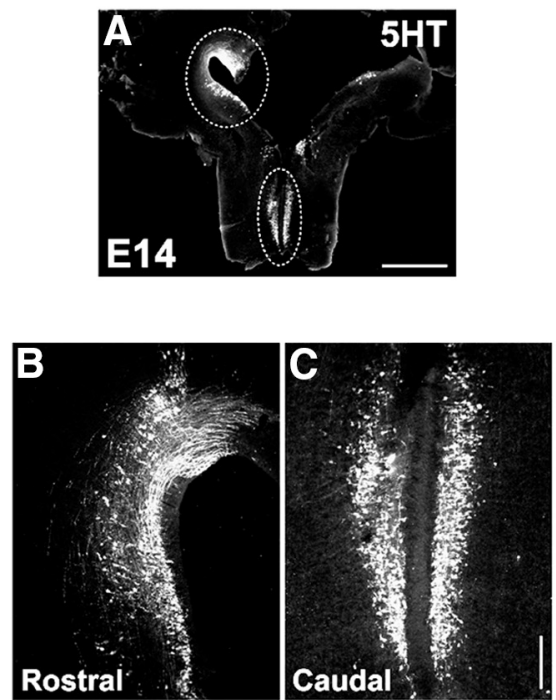

E

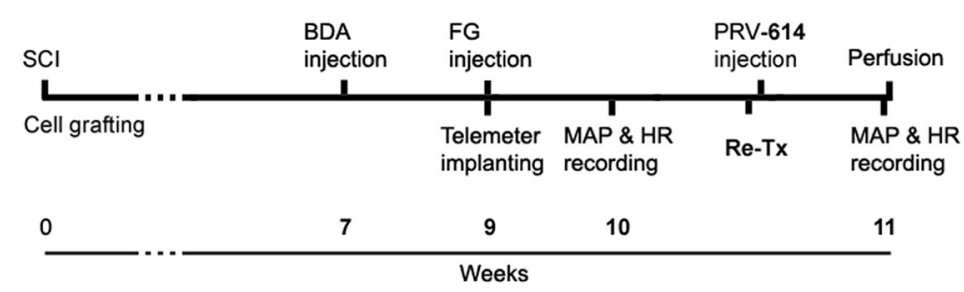

Figure 1. Schematics illustrate the experimental procedures. $A-C$, Donor cells are dissected from the RN in the GFP transgenic rat embryonic brainstem. Immunostaining for 5 -HT indicates $(\boldsymbol{A})$ the distribution of numerous early-stage serotonergic neurons in the rostral $(\boldsymbol{B})$ and caudal $(\boldsymbol{C})$ RN in an E14 brainstem. Dotted lined areas are approximately the regions dissected for cell grafting. D, A diagram represents cell transplantation and neuronal tract tracing. Immediately after spinal cord transection at T4 level (T4-Tx), E14 NSCs are implanted into the lesion site of the spinal cord. Animals survive for 10 weeks. Three weeks before death, BDA is injected into the RVLM of brainstem to anterogradely trace descending vasomotor pathways that regenerated into the graft. FG is injected intraperitoneally to retrogradely label SPNs in the spinal cord. RN-NSC-grafted rats received injections of PRV-614 into bilateral kidneys to retrogradely and transsysnaptically label neurons in the graft and host brainstem. E, A timeline shows the experimental arrangement. Post-g. N, Sympathetic postganglionic neurons. Scale bars: $A, 2 \mathrm{~mm} ; C, 200 \mu \mathrm{m}$.

growth factors to support graft survival as described previously for transplantation (Lu et al., 2012; Hou et al., 2013b), including BDNF (50 $\mu \mathrm{g} /$ $\mathrm{ml})$, neurotrophin-3 (50 $\mu \mathrm{g} / \mathrm{ml})$, platelet-derived growth factor-AA (10 $\mu \mathrm{g} / \mathrm{ml})$, insulin-like growth factor $1(10 \mu \mathrm{g} / \mathrm{ml})$, epidermal growth factor $(10 \mu \mathrm{g} / \mathrm{ml})$, basic fibroblast growth factor $(10 \mu \mathrm{g} / \mathrm{ml})$, glial cell linederived neurotrophic factor $(10 \mu \mathrm{g} / \mathrm{ml})$, and hepatocyte growth factor $(10 \mu \mathrm{g} / \mathrm{ml})$.

\section{Spinal cord surgery and cell transplantation}

The rats were anesthetized with isoflurane, and an incision of $2-3 \mathrm{~cm}$ was created in the skin of thoracic back. Connective tissues and muscles were moved aside with a tissue separator, and T3 dorsal laminectomy was performed. The dura was cut longitudinally in the midline. We recently reported that microaspiration in lesion surgical techniques for SCI caused severe fibrotic scarring and cavitation, which, in turn, resulted in poor survival and integration failure of grafted cells (Hou et al., 2018). In the present experiment, the spinal cord at T4 level was thus completely transected with iridectomy microscissors only. The dura was sutured before the cell injection to retain cells after the active bleed stopped. Embryonic RN/SC tissues were transplanted as small chucks immediately after injury. A total of $8 \mu \mathrm{l}$ of E14 RN or SC cells $\left(1.0 \times 10^{6} / \mu \mathrm{l}\right)$ were injected into the lesion site with a $10 \mu \mathrm{l}$ Hamilton syringe. This number was obtained by disassociating a comparable tissue sample to single-cell solution and counting under microscope. Afterward, musculature and skin were closed. Rats were given injections of lactated Ringer's solution, cefazolin $(10 \mathrm{mg} / \mathrm{kg})$, and Buprenex $(0.035 \mathrm{mg} / \mathrm{kg}$; Reckitt Benckiser) for care after surgery. Animals had their urinary bladder manually expressed at least 3 times daily for the first 2 weeks after surgery and then twice per day until death. Animals survived for an additional 10 weeks for cardiovascular measurement, tract tracing, or histological analysis.

\section{Anterograde, retrograde, and transsynaptic tract tracing}

To examine the potential regenerated host supraspinal vasomotor pathways, in one cohort of animals grafted with RN-NSCs $(n=5)$, biotinylated dextran amine (BDA, 10\% in distilled water, molecular weight 10,000 , Invitrogen) was bilaterally injected into the RVLM, one of major structures regulating cardiovascular function, to anterogradely label the descending axon terminals 7 weeks after cell grafting (3 weeks before perfusion) (Dampney et al., 2000). The coordination of injection relative to bregma was as follows: rostrocaudal $11.80 \mathrm{~mm}$, mediolateral $2.3 \mathrm{~mm}$, dorsoventral $7.6 \mathrm{~mm}$; rostrocaudal $12.30 \mathrm{~mm}$, mediolateral $2.2 \mathrm{~mm}$, dorsoventral $7.4 \mathrm{~mm} ; n=5$ in RN-NSC group) (Hou et al., 2013a). After the skull was opened using a drill, a total of $1.0 \mu \mathrm{l} /$ site was injected slowly with a Hamilton syringe. Buprenex $(0.035 \mathrm{mg} / \mathrm{kg})$ was administered subcutaneously after awake from anesthesia and on the following day to control postoperative pain.

FluoroGold (FG, $0.5 \% 200 \mu \mathrm{l}$ in distilled water) was intraperitoneally injected to retrogradely label SPNs in the intermediolateral cell column (IML) of thoracolumbar cord 1 week before perfusion (Akhavan et al., 2006). To verify the graft-relayed reconnection of supraspinal sympathetic pathways, we injected transsynaptic tracer pseudorabies virus (PRV-614) encoding red fluorescent protein (RFP) (Bartha strain, courtesy of Dr. Michael A. Lane) into the kidney in one cohort of rats grafted with RN-NSCs $(n=8)$ and a control group $(n=7)$ that received T4 spinal cord transection but no cellular graft (Schramm et al., 1993; Duale et al., 2009; Mironets et al., 2020). Approximately 10 weeks after cell 
transplantation/SCI, isoflurane-anesthetized rats received a retroperitoneal skin incision of $2-3 \mathrm{~cm}$ length on both sides to expose the kidney. A total of $6 \mu$ l PRV-614 $\left(10^{8} \mathrm{pfu} / \mathrm{ml}\right)$ was inoculated in the bilateral kidneys. The injections were performed in 3 sites of the renal parenchyma (1.0 $\mu \mathrm{l} / \mathrm{site})$ on the longitudinal midline of the convex surface of the kidney using a fine glass needle, filled with mineral oil and connected to a $10 \mu \mathrm{l}$ Hamilton syringe. Animals were perfused at 72 or $96 \mathrm{~h}(n=3$ or $n=4$ each) after viral injection. The spinal cord and brain were dissected for histology.

\section{Telemetric assessments of cardiovascular function}

A radio telemetric pressure transducer (Data Sciences International) was implanted in the abdomen to monitor cardiovascular parameters and AD 9 weeks following SCI/graft. Basal resting cardiovascular parameters, including mean arterial pressure (MAP) and heart rate (HR), and as well as naturally occurring or colorectal distension (CRD)-induced $\mathrm{AD}$, were measured 1 week later.

Telemeter implantation. Isoflurane-anesthetized rats were placed in a supine position. A skin incision was made in the midline of ventral abdomen, and the muscle layer was opened to expose the abdominal cavity. The organs were pushed aside, and the descending abdominal aorta was separated with two cotton swabs and fine forceps from the vein. Then, the catheter of a telemeter (model HD-S10, Data Sciences International) was tipped into the abdominal descending aorta for 3-4 cm. A drop of tissue glue was used to seal the lesion of artery. The transducer body was anchored on the abdominal wall. After organs were replaced to their original places, the muscle and skin layers were then sutured, respectively. Rats were given subcutaneous injections of Buprenex and cefazolin after surgery.

Basal hemodynamic recordings. The Dataquest Acquisition \& Analysis System ART software (Data Sciences International) was used for telemetric monitoring of hemodynamic parameters. Basal cardiovascular parameters, including resting MAP and $\mathrm{HR}$, were recorded in the following week after telemeter implantation. During the recording, pulse arterial pressure was transmitted as a radiofrequency signal to a receiver, data from receivers were collected and analyzed, and MAP and HR values were derived from the pulse arterial pressure with a computerized acquisition system. Briefly, a cage housing a single rat was placed on the top of the receiver for 10-15 min to calm the rat down and to stabilize cardiovascular parameters after turning the telemeter on. Then MAP and HR were then recorded for at least 30 min with sampling rate of $10 \mathrm{~s}$. Data were averaged for statistics.

Detecting naturally occurring $A D$. Similar to what we did previously (Mironets et al., 2018, 2020), the incidence of naturally occurring AD on the $24 \mathrm{~h}$ basal parameter recordings was automatically detected with an algorithm program written in MATLAB (The MathWorks). Concurrent values for MAP and $\mathrm{HR}$ were recorded at $2 \mathrm{~s}$ intervals for $24 \mathrm{~h} 1$ week after telemeter implantation. The software processed these waveforms to effectively estimate the number of events where an abnormally sharp MAP increase was accompanied by HR decrease (Rabchevsky et al., 2012). The signals were initially low-pass filtered at a $0.04 \mathrm{~Hz}$ cutoff frequency to smooth out transient spikes and limit their impact on threshold crossings. In consideration of strain variation of F344 rats, the conditions were recognized as naturally occurring $\mathrm{AD}$ as follows: the average value of MAP increase from the baseline $T p=15 \mathrm{mmHg}$ and $\mathrm{HR}$ decrease $T h=$ $15 \mathrm{bpm}$ within the MAP event interval. The parameters were chosen based on the offset of event to be 0 in naive rats. As a naturally occurring $\mathrm{AD}$ episode, the difference between the MAP peak and minimum values within the previous $T r=35 \mathrm{~s}$ must be $T s=\geq 20 \mathrm{mmHg}$.

$C R D$-induced $A D$. To initiate spinal viscero-sympathetic reflexes, 1 week after telemeter implantation, a latex balloon-tipped catheter (AA6110, Coloplast) was inserted for $\sim 2 \mathrm{~cm}$ into the colorectum and secured to the tail with tape (Maiorov et al., 1997). Animals were then placed into a restrain plastic tube and put on the receiver. Rats were allowed to habituate to adjust for at least $15 \mathrm{~min}$ to stabilize basal cardiovascular parameters. CRD was then induced by inflation of the balloon with $1.4 \mathrm{ml}$ of air for $1 \mathrm{~min}$ (exerting a pressure of $\sim 30 \mathrm{mmHg}$ ). The parameters of MAP and HR were measured before (baseline), during, and after (recovery) a 1 min period of balloon catheter inflation with sampling rate of $3 \mathrm{~s}$. An animal was regarded as dysreflexic if the distension produced a rise in MAP and a decrease in HR (Krenz and Weaver, 1998). At least two distensions with a 15-30 min recovery period were implemented in a quiet environment. The difference between the baseline and change in MAP and HR was calculated for each trial and then averaged over the two trials. In addition, the time of MAP recovery was measured. The recovery time was defined from deflation of the catheter balloon to the time point when the value of MAP descended to the average level before CRD. The values from two trials were averaged for statistics.

\section{Pharmacological interventions and spinal cord retransection}

To confirm specific effects of graft-derived 5-HT on hemodynamic improvements, pharmacological interventions were used for cardiovascular assessments. Previous studies demonstrated that $5-\mathrm{HT}_{2 \mathrm{~A}}$ receptors are expressed on autonomic neurons in the rat spinal cord and regulate cardiovascular function (Doly et al., 2004; Cormier et al., 2010). To examine whether hemodynamic recovery was related to the reestablished interaction of 5-HT and receptors, we delivered ketanserin, an antagonist of $5-\mathrm{HT}_{2 \mathrm{~A}}$ receptors, in rats grafted with RN-NSCs $(n=6)$ during the resting parameter and $\mathrm{CRD}$-induced $\mathrm{AD}$ tests. Animals receiving SCNSCs $(n=5)$ or injury only $(n=5)$ served as 2 controls. Three different dosages were subcutaneously administered, including $0.5,1.5$, and 3.0 $\mathrm{mg} / \mathrm{kg}$, with increasing dose pattern and at least $30 \mathrm{~min}$ intervals for recovery.

To verify RN-NSC graft-mediated cardiovascular improvements, we performed spinal cord retransection to interrupt graft-derived descending projections in one cohort of rats $(n=4)$ after cardiovascular functional assay at the 10th week. Isoflurane-anesthetized rats were reopened in skin and muscle layers to expose the injury/graft site of spinal cord at T4 level. After the dura was cut longitudinally in the midline, the spinal cord was completely retransected $2-3 \mathrm{~mm}$ caudal to the graft site with iridectomy microscissors. Finally, the muscle and skin were closed after the bleeding stopped. Rats were then given subcutaneous injections of Buprenex and cefazolin. One week after spinal cord retransection, resting cardiovascular recordings and $\mathrm{CRD}$-induced $\mathrm{AD}$ were processed again, as described before. At the conclusion of recordings, rats were intraperitoneally injected with a lethal dose of Euthasol for perfusion.

\section{Histological analysis}

Tissue processing and immunohistochemistry. Rats were transcardially perfused with $0.1 \mathrm{~m}$ PBS, $\mathrm{pH} 7.4$, followed by $4 \%$ PFA in PBS. The spinal cord and brain were dissected out and put in the same solution for postfixation overnight. Tissues were then transferred to $30 \%$ sucrose in $0.1 \mathrm{M}$ TBS for at least $24 \mathrm{~h}$. One $3 \mathrm{~cm}$ spinal cord segment containing the lesion site $0.5 \mathrm{~cm}$ rostral and $2.5 \mathrm{~cm}$ caudal to the lesion (approximate levels T2-T10) was embedded in gum tragacanth (Millipore Sigma) in $30 \%$ sucrose/PBS, whereas the brainstem was embedded in Tissue-Tek O.C.T. compound (Sakura Finetek) for cryostat, as described previously (Cameron et al., 2006). The spinal cord or brainstem was serially cryosectioned in the longitudinal, horizontal plane at $35 \mu \mathrm{m}$ in 6 series of free-floating sections. For immunostaining, free-floating sections were blocked in TBS containing $0.25 \%$ Triton X and 5\% normal donkey serum for $1 \mathrm{~h}$, and then incubated with primary antibodies (Table 1), including GFP to label GFP-expressing embryonic cells, GFAP to label astrocytes, NeuN to label mature neurons, 5-HT or tryptophan hydroxylase 1 (TPH1) to label serotonergic neurons and axons, ChAT to label cholinergic neurons, dopamine- $\beta$-hydroxylase $(\mathrm{DBH})$ to label noradrenergic neurons, synaptophysin to label presynaptic terminals, platelet-derived growth factor receptor- $\beta$ (PDGFR- $\beta$ ) to label blood vessel-derived fibroblasts, collagen III (Col-III) to label collagen, CSPG to label astroglial inhibitory factors, vascular endothelial growth factor receptor 2 (VEGFR2) to label blood vessels, and PRV and RFP for infected cells by PRV-614. Sections were incubated in primary antibody solution overnight at $4^{\circ} \mathrm{C}$ and then incubated in AlexaFluor-488-, AlexaFluor-594-, or AlexaFluor-647-conjugated goat or donkey secondary antibodies (1:500; Invitrogen) for $3 \mathrm{~h}$ at room temperature. Notably, different combinations of immunostaining for multiple antibodies were performed for different observations. To label $5-\mathrm{HT}^{+}$neurons or BDA ${ }^{+}$ 
Table 1. Information of antibodies

\begin{tabular}{llll}
\hline Antibody & Host & Working concentration & Company \\
\hline GFP & Chicken & $1: 1000$ & Invitrogen \\
& Goat & $1: 1500$ & Millipore \\
GFAP & Mouse & $1: 1500$ & Millipore \\
& Rabbit & $1: 1500$ & Dako \\
NeuN & Mouse & $1: 200$ & Millipore \\
5-HT & Rabbit & $1: 1000$ & ImmunoStar \\
TPH1 & Rabbit & $1: 1000$ & Novus \\
ChAT & Goat & $1: 1000$ & Millipore \\
DBH & Mouse & $1: 500$ & Millipore \\
Synaptophysin & Mouse & $1: 1000$ & Millipore \\
PDGFR- $\beta$ & Rabbit & $1: 1000$ & Abcam \\
Col-III & Mouse & $1: 1000$ & Abcam \\
CSPG & Mouse & $1: 100$ & Sigma Millipore \\
VEGFR2 & Mouse & $1: 1000$ & Millipore \\
PRV & Rabbit & $1: 10,000$ & courtesy of Dr. Lynn Enquist \\
RFP & Rabbit & $1: 1000$ & Rockland \\
& Mouse & $1: 1000$ & Rockland \\
\hline
\end{tabular}

fibers in the graft for light-level microscopy, endogenous peroxidase activity was blocked with $0.6 \%$ hydrogen peroxide in TBS for $15 \mathrm{~min}$. After washing for three times, sections were incubated in avidin-biotinylated peroxidase complex (Elite kit; 1:100; Vector Laboratories) for $6 \mathrm{~h}$ at room temperature. DAB $(0.05 \%)$ was used as chromagen, with reactions sustained for $0.5-10 \mathrm{~min}$. Further, the sections were processed for GFP/ GFAP double immunofluorescent staining to visualize the area of graft or host tissue. After being mounted on a glass slide, the sections were finally coverslipped with mounting medium containing DAPI, except those labeled with FG. The slides were then reviewed and imaged using a fluorescent microscope (DM5500B, Leica Microsystems), connected to a digital camera (C11440, Hamamatsu) and installed with a Slidebook 6 software (Intelligent Imaging Innovations), and a confocal microscope (TCS SP8, Leica Microsystems).

Quantification of immunolabeling. All quantifications for histological analysis were conducted by an observer blinded to group identity. To determine the number of 5- $\mathrm{HT}^{+}$cells in the graft, one serial of horizontal longitudinal thoracic spinal cord sections triple-labeled for 5-HT/GFP/ GFAP was examined under fluorescence microscope. 5-HT/GFP doublelabeled cells were counted at $100 \times$ magnification in two sections containing the IML region and one section containing the central canal in each animal. Only cells displaying a neuronal profile with cellular processes were counted. The total number of labeled neurons in all three sections was added together and used for statistical analysis. We counted $5-\mathrm{HT}^{+}$fibers initiating from $\mathrm{GFP}^{+}$grafts and projecting into the IML region in two sections containing FG retrogradely labeled SPNs per animal, with which the dense $5-\mathrm{HT}^{+}$fibers were often associated. The quantification was performed at $400 \times$ magnification using epifluorescent illumination and appropriate wavelength filters. To standardize the ROI for every animal, two levels were selected in each section to quantify $5-\mathrm{HT}^{+}$axons. One was $0.2 \mathrm{~mm}$ caudal to the graft, a short distance for NSC-derived axons, whereas the other was $5.0 \mathrm{~mm}$ for the projections with a long distance. Using a calibrated eyepiece with a scale in the objective of scope, we made a vertical line across the IML and counted the number of $5-\mathrm{HT}^{+}$fibers that crossed the line. For each animal, a total number of $5-\mathrm{HT}^{+}$axons were obtained by summing labeled fibers on two individual sections. The distance of longest serotonergic fibers in the IML was also measured using a calibrated eyepiece with a scale at $200 \times$ magnification. For BDA-labeled medulla-derived projections, the number and longest distance of visible profiles within the graft were quantified at $200 \times$ magnification under light microscopy. The total number of BDA-labeled axonal profiles and the averaged longest distance of fibers in all sections were used for statistical analysis. In transsynaptic neuronal tracing, injection of PRV-614 into the kidney of rats transplanted with RN-NSCs labeled neurons within the graft and host brainstem at different time courses. The number of PRV-labeled grafted neurons and labeled grafted serotonergic neurons, identified by PRV-614/GFP double immunolabeling or PRV-614/GFP/5-HT triple immunolabeling, were counted within the lesion/graft area in one serial of sections. In a set of sections from RN-NSC-grafted rats and injury controls, PRV-labeled host neurons in the brainstem were also counted. The number of labeled neurons in each section were added together per animal for statistics.

\section{Statistics}

Data of cardiovascular parameters among four groups were analyzed by one-way ANOVA with Fisher's PLSD. The comparison of hemodynamics before and after pharmacological interventions was processed with repeated-measures ANOVA followed by post hoc with Bonferroni corrections, whereas that before and after spinal cord retransection was compared with paired $t$ test. Histological data were analyzed using two-way ANOVA or unpaired $t$ test according to data types. A significance criterion of $p<0.05$ was used. All values are expressed as the mean \pm SEM.

\section{Results}

Adult inbred F344 rats underwent T4 complete spinal cord transection and cell grafting into the lesion site. Donors were E14 RN-NSCs or SC-NSCs from the same strain of GFP transgenic rats. An implanted telemetric system was used to evaluate cardiovascular functional recovery, including resting MAP and HR, naturally occurring or CRD-induced AD after 10 weeks. Selected animals underwent pharmacological interventions or spinal cord retransection below the graft to examine relayed serotonergic regulation of hemodynamics. Multiple neuronal tracers, such as BDA, FG, and PRV-614, were injected to identify potentially reestablished supraspinal vasomotor pathways (Fig. $1 D, E$ ).

\section{Cellular grafts survive and partially integrate into the host spinal cord}

Rats received T4 spinal cord transection and immediate transplantation of either $\mathrm{GFP}^{+}$E14 RN-NSCs or SC-NSCs into the spinal lesion. Some animals received an injury but not a transplant. To examine grafted cell survival and integration, animals were perfused 10 weeks after SCI and the spinal cords were processed for histological analysis. $\mathrm{GFP}^{+} \mathrm{RN}$-or SC-NSC implants survived well in the majority of animals ( $\sim 70 \%-80 \%$; Fig. $2 A)$. In these animals, the transplants expanded and integrated into the host gray and white matter in both the rostral and caudal directions. GFP ${ }^{+}$cells were found through the length of the lesion site and bridged the rostral and caudal stumps of spinal cord (Fig. 2A-D).

As expected, injury itself results in an astrocytic glial scar within the lesion penumbra (Fig. $2 F$ ) and a fibrotic scar located with the lesion site (Fig. 2E,F) Moreover, this tissue was rich in CSPGs and Col-III (Fig. 2G). In animals that had NSCs transplanted into the lesion site, there was usually a scar-like tissue that encroached the center of the graft from the lateral edges of the lesion site. Immunostaining revealed that this region contained many PDGFR $-\beta^{+}$fibroblasts and virtually no $\mathrm{GFP}^{+}$cells. A few PDGFR- $\beta^{+}$cells were also observed in $\mathrm{GFP}^{+}$grafts, where they formed tube-like structures, suggesting their participation in the genesis of nascent blood vessels (Fig. 2B). Previous reports reported that fibroblasts in the scar tissue are mainly derived from pericytes of blood vessels in the neighboring spinal cord parenchyma (Göritz et al., 2011; Soderblom et al., 2013). Accordingly, as this type of fibroblast predominantly produces Col-III, we found intense accumulation of Col-III in the fibrotic scar. Notably, deposition of Col-III overlapped with PDGFR- $\beta$-labeled fibroblasts in the scar but not with those in the graft (Fig. 2C). In parallel, there was appreciable GFAP expression at the host/graft interface in the cords grafted with NSCs. CSPGs were present within the glial scar in the lesion penumbra as well as the fibrotic 

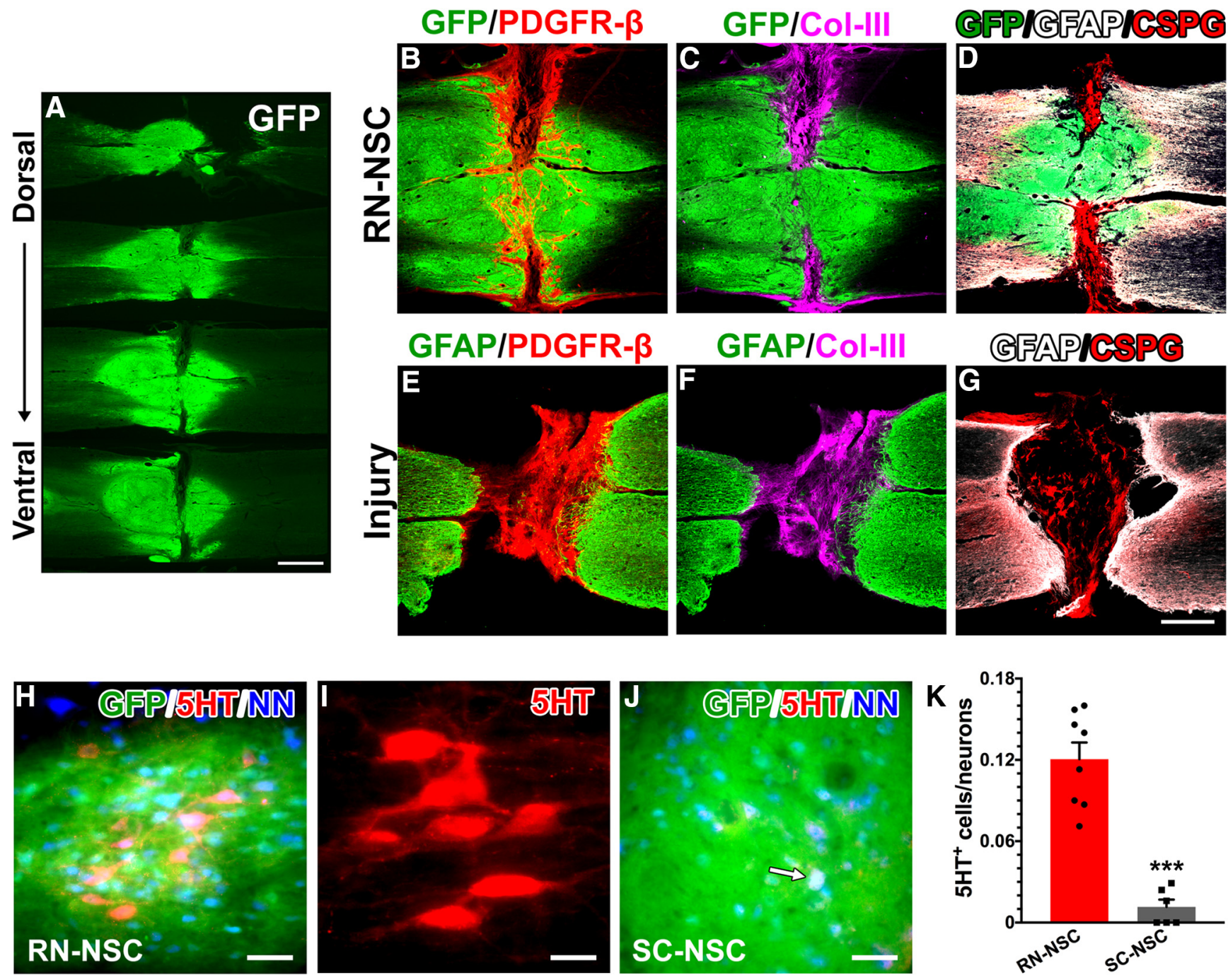

Figure 2. Grafted E14 BS-NSCs partially integrate into the spinal cord. $A$, Surviving GFP ${ }^{+}$BS-NSC implants are shown in a series of longitudinal spinal cord sections from the dorsal to ventral. Grafts form a bridge in the middle part and connect the rostral and caudal cords. $\boldsymbol{B}-\boldsymbol{G}$, Immunostaining revealed that (B) numerous PDGFR- $\beta^{+}$fibroblasts are present in the portions of the lesion devoid of GFP ${ }^{+}$tissue. Col-III ( $(\boldsymbol{C})$ and CSPG $(\boldsymbol{D})$ are heavily deposited in this region. There is a little expression of GFAP at the interface of host/graft. In injury controls $(\boldsymbol{E}-\boldsymbol{G})$, however, a dense GFAP ${ }^{+}$ glial scarring is often present at the stumps of spinal cord. Intense Col-III and CSPG are stained into the lesion site of spinal cord. $\boldsymbol{H}-\boldsymbol{K}$, Abundant serotonergic neurons grow in RN-NSC grafts $(\boldsymbol{H}, \boldsymbol{I})$, whereas very few are detected (arrow) in SC-NSC grafts $(J)$. These neurons express NeuN, indicating that they have developed mature. $\boldsymbol{K}$, Cell quantification demonstrated that the percentage of serotonergic neurons in RN-NSC grafts is significantly higher (unpaired $t$ test, ${ }^{* * *} p<0.001$ ) than those in SC-NSC grafts ( $n=6$ or 8 per group). Scale bars: $\boldsymbol{A}, 2 \mathrm{~mm} ; \mathbf{G}, 0.5 \mathrm{~mm} ; \boldsymbol{H}, J, 50 \mu \mathrm{m} ; \boldsymbol{I}$, $20 \mu \mathrm{m}$.

scar described above (Fig. 2D). Thus, RN-NSC and SC-NSC implants survived and partially integrated into the host spinal cord.

Immunostaining for 5-HT revealed many serotonergic neurons within the RN-NSC grafts. These neurons expressed NeuN, a marker of mature neurons (Fig. $2 H, I$ ). Significantly fewer $5-\mathrm{HT}^{+}$cells were detected in SC-NSC grafts (Fig. $2 \mathrm{~J}$ ). These cells could be derived from the few serotonergic neurons within the spinal cord itself (Mouchet et al., 1986; Newton and Hamill, 1988) or from a contamination by the caudal brainstem during embryo dissection, as previously described (Hou et al., 2013b). Quantitative analysis illustrated that the percentage of $\mathrm{NeuN}^{+}$ neurons that were also $5-\mathrm{HT}^{+}$is much higher within RN-NSC grafts $(12 \pm 1.2 \%)$ than in SC-NSC grafts $(1.2 \pm 0.5 \%$; unpaired $t$ test, $p<0.001$; Fig. $2 K)$. In consideration of $\sim 15 \%$ of $5-\mathrm{HT}^{+}$ neurons in the caudal raphe nucleus (Charnay and Léger, 2010), this is a very high percentage. No 5-HT labeling was observed within the lesion cavity of nontransplanted cords. The results support the notion that a greater number of progenitors derived from the $\mathrm{RN}$ differentiate into serotonergic neurons than those harvested from the spinal cord, although both grafts contained some degree of $5-\mathrm{HT}^{+}$neurons.

\section{Grafted $5-\mathrm{HT}^{+}$neurons project to the caudal}

autonomic region

Cells originating from both types of embryonic NSC grafts extended numerous $\mathrm{GFP}^{+}$processes toward the caudal spinal cord (Fig. $3 A, B$ ), consistent with previous observations (Hou et al., $2013 \mathrm{~b}$ ). In the cords grafted with RN-NSCs, many $5-\mathrm{HT}^{+}$axons extended caudally over several spinal segments (Fig. $3 A$ ). These $5-\mathrm{HT}^{+}$fibers colocalized with GFP, indicating that they originated from the implants (Fig. $3 D-F$ ). Interestingly, many of these $\mathrm{GFP}^{+} / 5-\mathrm{HT}^{+}$fibers projected toward FG-labeled SPNs in the bilateral IML regions (Fig. 3D-G,I). Likewise, dense $\mathrm{GFP}^{+} / 5-$ $\mathrm{HT}^{+}$fibers distributed in the caudal gray commissure around the central canal, another autonomic region in the spinal cord (Fig. $3 J, K)$. In contrast, significantly fewer $5-\mathrm{HT}^{+}$fibers were ob- 

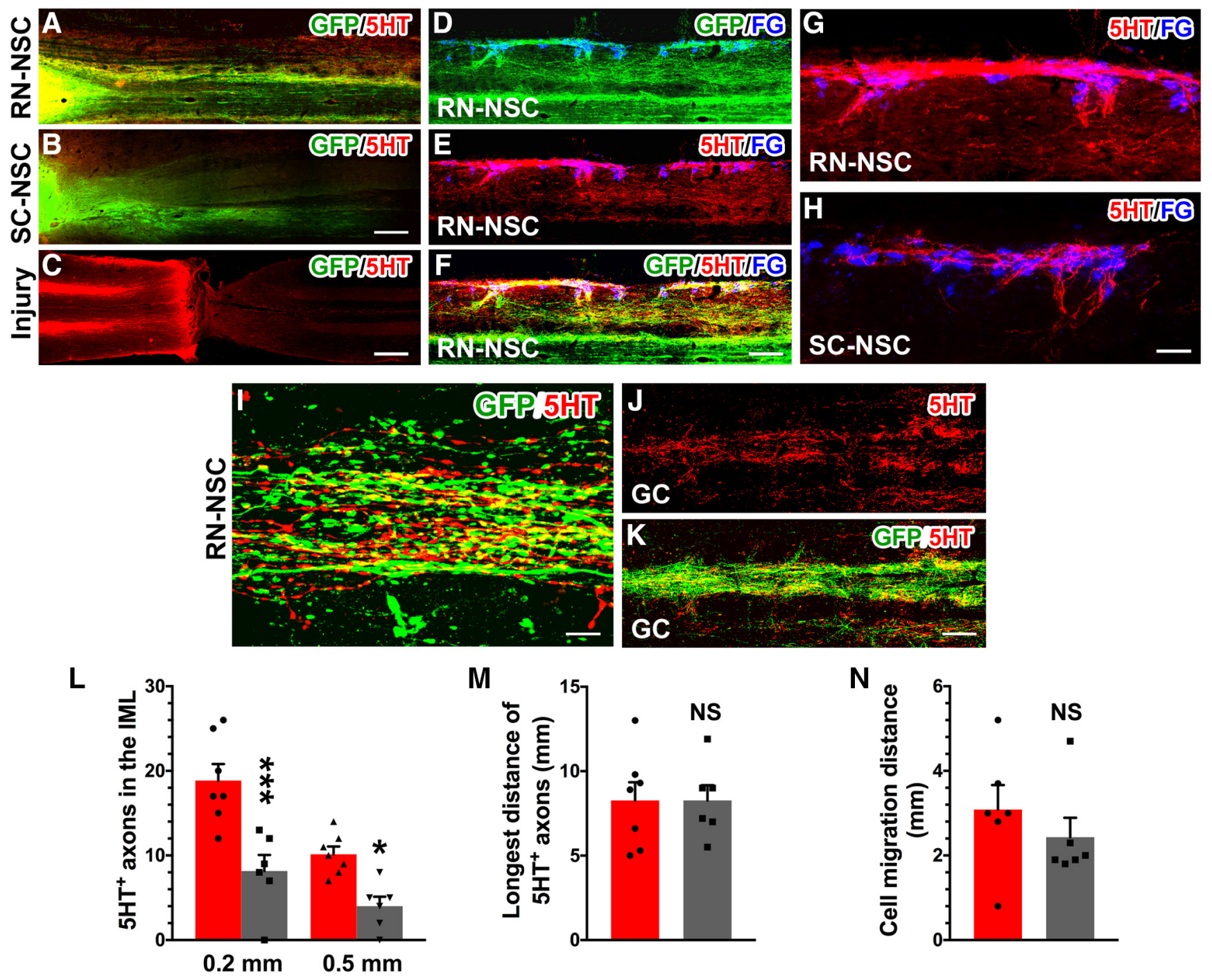

M

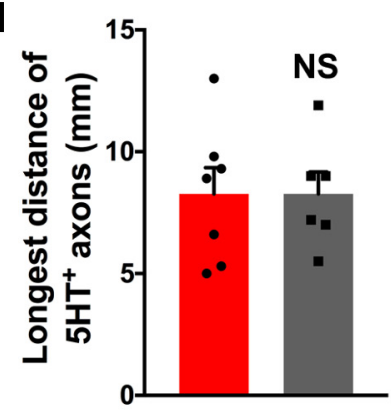

N

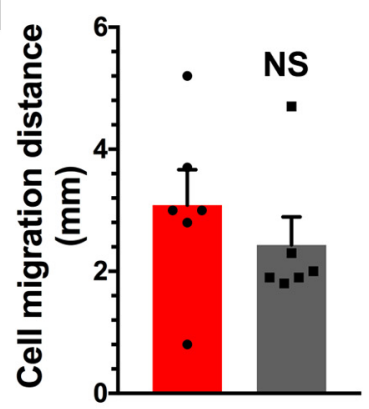

\section{RN-NSC}

Figure 3. RN-NSC grafts derive abundant serotonergic fibers and project long distance to the caudal autonomic regions. $\boldsymbol{A}-\boldsymbol{C}$, Both grafted ( $\boldsymbol{A})$ RN-NSCs and ( $\boldsymbol{B})$ SC-NSCS initiate numerous GFP ${ }^{+}$ extensions toward the distal spinal cord while the lesion interrupts neuronal pathways in ( $\boldsymbol{C}$ injury controls, indicated by disrupted 5 - $H T^{+}$serotonergic fibers $(n=6$ or 8 per group). $\boldsymbol{D}-\boldsymbol{H}$, In the spinal cord grafted with RN-NSCs $(\boldsymbol{D}-\mathbf{G})$, dense 5 - $\mathrm{HT}^{+}$fibers are overlapped with GFP and spanned several segments to the caudal IML, innervating to FG-labeled SPNs. In contrast, much less 5 -HT ${ }^{+}$ fibers are observed in SC-NSC grafted cords $(\boldsymbol{H})$. I, Colocalization of $5-\mathrm{HT}^{+} / \mathrm{GFP}^{+}$axons in the IML caudal to the lesion is shown under a higher magnification. $\boldsymbol{J}, \boldsymbol{K}$, Ample $\mathrm{GFP}{ }^{+} / 5$ - $\mathrm{HT}^{+}$fibers distribute in the caudal gray commission (GC) around the central canal. $\boldsymbol{L}-\mathbf{N}$, Axonal quantification demonstrated that $(\boldsymbol{L})$ a greater number of serotonergic fibers emerged from RN-NSC grafts than those from SC-NSC grafts (two-way ANOVA followed by Tukey's adjustment: ${ }^{*} p<0.05$; ${ }^{* *} p<0.001$ between groups), although the longest distance of 5 -HT ${ }^{+}$fibers ( $\boldsymbol{M}$ ) had no difference between the two groups (unpaired $t$ test, $p>0.05$ ), $(\boldsymbol{N})$ nor did the cell migration from the edge of spinal cord stumps (unpaired $t$ test, $p>0.05)$. NS, not significant. Scale bars: $\boldsymbol{B}, 200 \mu \mathrm{m}$; C, $400 \mu \mathrm{m} ; \boldsymbol{F}, 150 \mu \mathrm{m} ; \boldsymbol{H}, 50 \mu \mathrm{m} ; \boldsymbol{I}, 12 \mu \mathrm{m} ; \boldsymbol{K}, 100 \mu \mathrm{m}$.

served caudal to the SC-NSC grafts (Fig. $3 \mathrm{~B}, \mathrm{H}$ ). 5 - $\mathrm{HT}^{+}$profiles were rarely detected below the lesion in the injury, nontransplanted control (Fig. 3C). Quantification of 5- $\mathrm{HT}^{+}$axons in the IML demonstrated that a greater number of serotonergic fibers emerged from RN-NSC grafts (from the graft/host interface: 0.2 $\mathrm{mm}, 18.9 \pm 1.9 ; 5.0 \mathrm{~mm}, 10.1 \pm 0.9)$ than those from SC-NSC grafts $(0.2 \mathrm{~mm}, 8.2 \pm 1.9 ; 5.0 \mathrm{~mm}, 4.0 \pm 1.1$; two-way ANOVA followed by Tukey's adjustment: ${ }^{\star} p<0.05 ;{ }^{* * *} p<0.001$ between groups) (Fig. $3 L$ ). There was no difference (unpaired $t$ test, $p>$ 0.05 ) in the length of the longest $5-\mathrm{HT}^{+}$fiber between the two groups (RN-NSC $8.3 \pm 1.1$ vs SC-NSC $8.3 \pm 0.9 \mathrm{~mm}$; Fig. $3 M$ ), nor the extent of grafted cell migration from the edge of the spinal cord stumps (RN-NSC $3.1 \pm 0.6$ vs SC-NSC $2.4 \pm 0.4 \mathrm{~mm}$; Fig. $3 N)$. Confocal $3 \mathrm{D}$ reconstructions demonstrate colocalization of GFP, 5-HT, and the presynaptic marker synaptophysin sur- rounding a neuron within the IML, suggesting that these axons formed putative synapses upon neurons in this autonomiccentric region (Fig. 4).

Host supraspinal vasomotor pathways regenerate into RN-NSC grafts

Transplanted NSCs that differentiate into neurons can help form a novel, compensatory circuit that relays information from supraspinal neurons to spinal neurons below the injury (Bonner and Steward, 2015; Assinck et al., 2017). To investigate whether supraspinal populations normally associated with regulating sympathetic function grow into the RN-NSC transplants, BDA was injected into the RVLM, one of the brainstem vasomotor centers, bilaterally 3 weeks before death (i.e., 7 weeks after SCI and transplantation). Histological analysis demonstrated that BDA- 

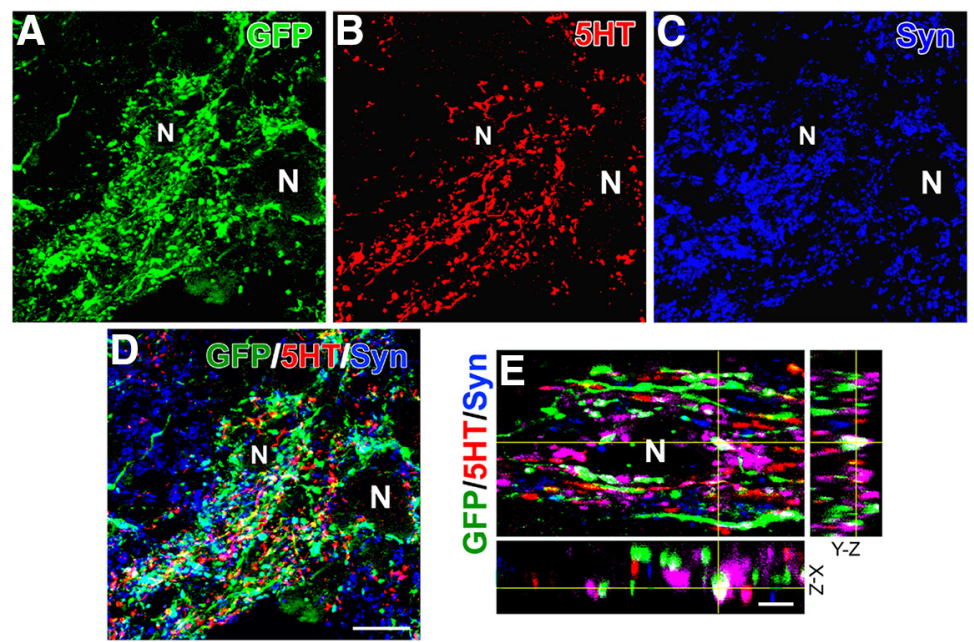

Figure 4. RN-NSC graft-derived serotonergic fibers form synapses in the caudal autonomic region. $\boldsymbol{A}-\boldsymbol{D}$, Confocal analysis demonstrates that numerous $\mathrm{GFP}^{+} / 5-\mathrm{HT}^{+}$axon terminals innervate to the IML, where the bouton-like structures form plentiful synapses onto adjacent neurons ( $N$ ), identified by expressing the presynaptic marker synaptophysin. $\boldsymbol{E}$, A higher magnification in 3 dimensions confirms established synapses (white). Scale bars: $\boldsymbol{D}, 20 \mu \mathrm{m} ; \boldsymbol{E}, 5 \mu \mathrm{m}$.
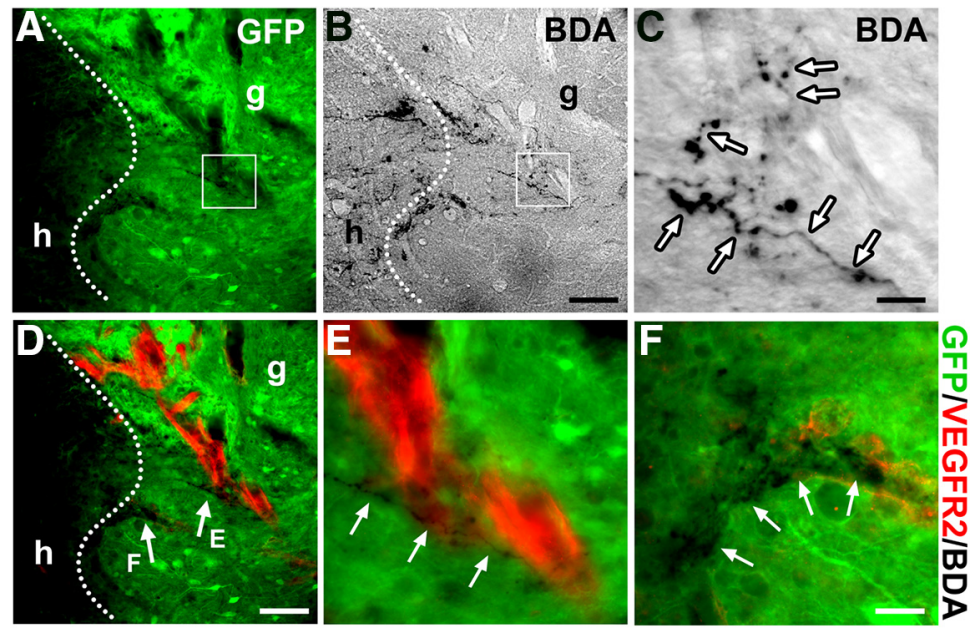

Figure 5. Descending vasomotor pathways regenerate into NSC grafts. $\boldsymbol{A}-\boldsymbol{C}$, BDA injection into the RVLM labels regenerated supraspinal axon terminals within the GFP ${ }^{+}$RN-NSC-grafted region in the spinal cord $(n=5)$. Dotted lines indicate the interface of host (h)/graft $(\mathrm{g})$. Labeled descending axon terminals (arrows) mainly emerge in the rostral part of GFP ${ }^{+}$grafts. $\boldsymbol{C}$, Higher magnification of the boxed region in $\boldsymbol{A}$ and $\boldsymbol{B}$. D-F, Costaining of BDA and immunofluorescent labeling of VEGFR-2 disclose that penetrated axon terminals often apposite along nascent blood vessels in GFP ${ }^{+}$implants. Scale bars: $\boldsymbol{B}, \boldsymbol{D}, 100 \mu \mathrm{m} ; \boldsymbol{C}, 12.5 \mu \mathrm{m} ; \boldsymbol{F}$, $30 \mu \mathrm{m}$.

labeled RVLM projections extended to the graft/host interface, with some of them traversing across the border into RN-NSC grafts (Fig. $5 A-C$ ). The majority of $\mathrm{BDA}^{+}$fibers that grew into the $\mathrm{GFP}^{+}$graft were located in the rostral portion of the transplant; very few were found in the epicenter. No BDA-labeled axon terminals extended beyond the length of the graft into the distal spinal cord. Quantitative analysis showed a total of $75 \pm 12$ $\mathrm{BDA}^{+}$axons within the graft. Interestingly, immunostaining revealed that BDA-labeled axon terminals that penetrated the cellular graft often aligned with blood vessels labeled with VEGFR2 (Fig. 5D-F). This suggests the importance of adequate nutrition supply or the role of endothelial cells in local environment for axon growth. These results are similar to our previous findings where host axon regeneration into grafts of whole brainstemderived NSCs or SC-NSCs was limited (Hou et al., 2013b). Nevertheless, this short-distance host axon regrowth may be sufficient for the formation of a compensatory relay circuit.
Transsynaptic tracing labels neurons in the graft and host brainstem

To more directly assess whether cells within the RN-NSC transplant are integrated into a novel circuit that may relay information from supraspinal neurons to sympathetically associated neurons caudal to the SCI, the retrograde, transsynaptic tracer PRV-614 was injected into the kidneys. At $72 \mathrm{~h}$ after viral inoculation, immunolabeling showed that numerous FG-labeled SPNs in the IML of spinal cord were infected with PRV (Fig. 6A,B). Some infected interneurons, which were not labeled with FG, were observed more medially in gray matter (Fig. 6B). Considering their proximity to $\mathrm{FG}^{+} \mathrm{SPNs}$, these interneurons are likely synaptically linked to the SPNs. PRV-labeled cells were found throughout the $\mathrm{GFP}^{+}$RN-NSC grafts, although the majority of them were located close to the caudal interface. Importantly, costaining for 5-HT antibody verified that $23 \pm 5.2 \%$ of PRV-infected neurons in the graft are serotonergic (Fig. $6 F-H)$. There were infected cells in the graft at both 72 and $96 \mathrm{~h}$ after inoculation (Fig. 6C,D,E). It did not show a difference in the number of PRV-infected neurons within the graft between these two survival times (Fig. 6I).

PRV transsynaptic tracing labeled neurons in the host brainstem. Although previous investigations did not support the likelihood of tracer transportation through central parasympathetic pathways in the kidney (Norvell and Anderson, 1983; Cano et al., 2004), it could not rule out the inappropriate evaluation resulting from insufficient expertise or technical limitations. To determine whether PRV-labeled neurons in the brainstem were infected through reestablished sympathetic pathways, we also injected PRV into the kidneys of a separate cohort of animals that received SCI but no graft so that their supraspinal regulation of renal sympathetic pathways was largely interrupted but parasympathetic pathways kept intact. In both the RN-NSC-grafted or injury-only animals, immunostaining for RFP revealed a few PRV-infected neurons in the dorsal motor nucleus of vagus nerve (DMV) and the nucleus ambiguus (NA), two preganglionic parasympathetic nuclei in the brainstem at $72 \mathrm{~h}$ transportation. Labeling was more apparent in these regions at $96 \mathrm{~h}$ after infection (Fig. 7A-H). The majority of RFP-labeled neurons here were costained with ChAT, indicating that they were cholinergic. In the DMV, PRV-infected neurons were adjacent to the nucleus tractus solitarius (NTS), which contained $\mathrm{DBH}^{+}$ noradrenergic neurons (Fig. $7 \mathrm{~B}, \mathrm{C}$ ). In addition, we observed a few PRV-infected cells colabeled with DBH in the NTS, which might indicate the short-distance projections from the NTS to DMV. PRV labeling in the parasympathetic nuclei was similar between RN-NSC-grafted and injury control rats, suggesting 

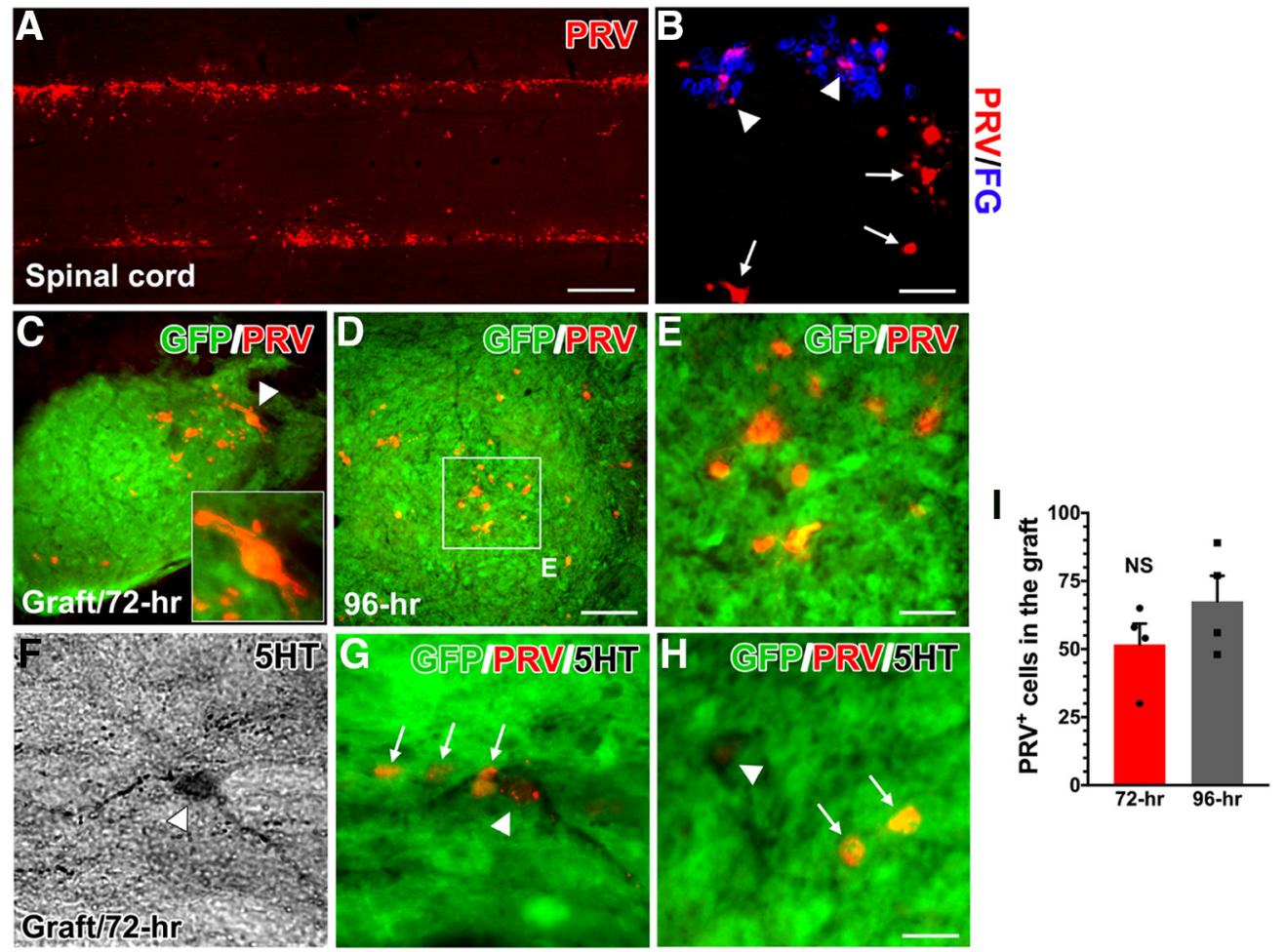

Figure 6. PRV transsynaptic tracing labels neurons in the graft. Ten weeks after cell transplantation, PRV-614 was injected into the bilateral kidneys to transsynaptically trace reestablished supraspinal sympathetic pathways in RN-NSC-grafted rats $(n=8) \cdot \boldsymbol{A}, \boldsymbol{B}$, At 72 h after inoculation $(n=4)$, immunolabeling shows that $(\boldsymbol{A})$ numerous PRV-infected SPNs emerge in the IML of caudal spinal cord, which are $(\boldsymbol{B})$ colabeled by FG (arrowheads). Some viral-infected neurons, but not FG-labeled (arrows), are observed in the middle area of gray matter, indicating the possibility of interneuronal identity linked to the SPNs. C-E, PRV injection transfects RN-NSCs in the grafts after $72(\boldsymbol{C})$ or $96 \mathrm{~h}(\boldsymbol{D}, \boldsymbol{E})(n=4)$ proliferation and transportation times. $\boldsymbol{C}$, Inset, A PRV-labeled neuron (arrowhead) in the graft with high magnification. $\boldsymbol{F}-\boldsymbol{H}, \mathbf{I m p o r t a n t l y , ~} 5$-HT antibody costaining verifies that a portion of PRV-infected neurons in the graft are serotonergic. $\boldsymbol{I}$, Statistical analysis shows that the number of PRV-infected neurons in the graft is not significantly (NS) (unpaired $t$ test, $p>0.05$ ) different from 72 to $96 \mathrm{~h}$ after injection. This suggests that grafted RN-NSCs rebuild descending autonomic regulation pathways following $S C l$ by forming synaptic connectivity with spinal SPNs. Scale bars: $A, 1.7 \mathrm{~mm} ; \boldsymbol{B}, 75 \mu \mathrm{m} ; \boldsymbol{D}, 80 \mu \mathrm{m} ; \boldsymbol{E}, \boldsymbol{H}, 20 \mu \mathrm{m}$.

that the tracer injected in the kidney can transsynaptically infect neurons within the brain following SCI via parasympathetic pathways.

However, there was a stark difference when we examined PRV infection of neurons in sympathetic premotor brainstem regions. We observed PRV-infected neurons in the RVLM, C1, and caudal $\mathrm{RN}$ only in RN-NSC-grafted rats $96 \mathrm{~h}$ after infection. PRVinfected neurons in the RVLM did not immunostain for DBH (Fig. 7I), indicating that they were not noradrenergic. PRVinfected neurons in the $\mathrm{C} 1$ region were $\mathrm{DBH}^{+}$(Fig. 7J). In the caudal RN, most infected neurons colabeled with a 5-HT antibody, indicating that they were serotonergic (Fig. $7 \mathrm{~K}, L$ ). We saw PRV-infected sympathetic premotor neurons in the host brainstem of all RN-NSC-grafted rats at $96 \mathrm{~h}$ viral transportation (Fig. $70)$. On the contrary, we did not observe any infected cells in these presympathetic regions in injured rats with no graft. These data indicate that these PRV-labeled neurons were infected via sympathetic but not parasympathetic circuitry.

Collectively, these data demonstrate that grafted RN-NSCs, including those that differentiated into $5-\mathrm{HT}^{+}$neurons, projected caudally and formed functional synapses upon SPNs within the IML and/or sympathetically associated interneurons. In turn, neurons in the supraspinal autonomic centers synapsed upon grafted RN-NSCs. Given our finding that supraspinal populations (e.g., RVLM) can regenerate into the RN-NSC graft (Fig. 5 ), these data illustrate that grafted RN-NSCs provide a means to relay transmission from supraspinal centers across the lesion to regulate sympathetic activity and improve cardiovascular function after SCI.

\section{Grafting RN-NSCs improves resting blood pressure}

A lasting hypotension and compensatory tachycardia at rest often occur after SCI at high levels. This has been attributed to interrupted supraspinal control of sympathetic tone. Notably, central serotonergic regulation is critical for normal, basal hemodynamics (Calaresu and Yardley, 1988). We found that grafting RNNSCs into the injured cord improves resting hemodynamic performance. Ten weeks after complete T4 transection, rats without any type of grafts had a lower resting MAP $(101 \pm 2 \mathrm{mmHg})$ and higher HR (459 $\pm 11 \mathrm{bpm})$, compared with naive, uninjured rats (MAP, $118 \pm 2 \mathrm{mmHg}$; HR, $372 \pm 6 \mathrm{bpm}$; MAP: one-way ANOVA, $p=0.005$, Fisher's PLSD, $p<0.01$; HR: ANOVA, $p=$ 0.001 , Fisher's PLSD, $p<0.001)$. However, the resting MAP in injured rats transplanted with RN-NSCs $(115 \pm 4 \mathrm{mmHg})$ was similar to that in naive controls, although the resting HR was still higher (458 $\pm 14 \mathrm{bpm}$, Fisher's PLSD, $p<0.001)$. In contrast, transplantation of E14 SC-NSCs, which are virtually devoid of $5-\mathrm{HT}^{+}$neurons, did not affect injury-induced hypotension or tachycardia. We observed a similar decrease in MAP and increase in HR in these animals compared with injured, nontransplanted control animals (both $p>0.05$ vs injury controls; both $p<0.05$ vs naive rats) (Fig. 8), consistent with our previous report (Hou et al., 2013b). These data indicate that neural grafts enriched with serotonergic neurons attenuate high-level SCI-induced hypotension.

\section{Grafting embryonic NSCs alleviates naturally occurring and} CRD-induced AD

Ten weeks after SCI and cell transplantation, continuous telemetric recordings were performed to determine the frequency of 

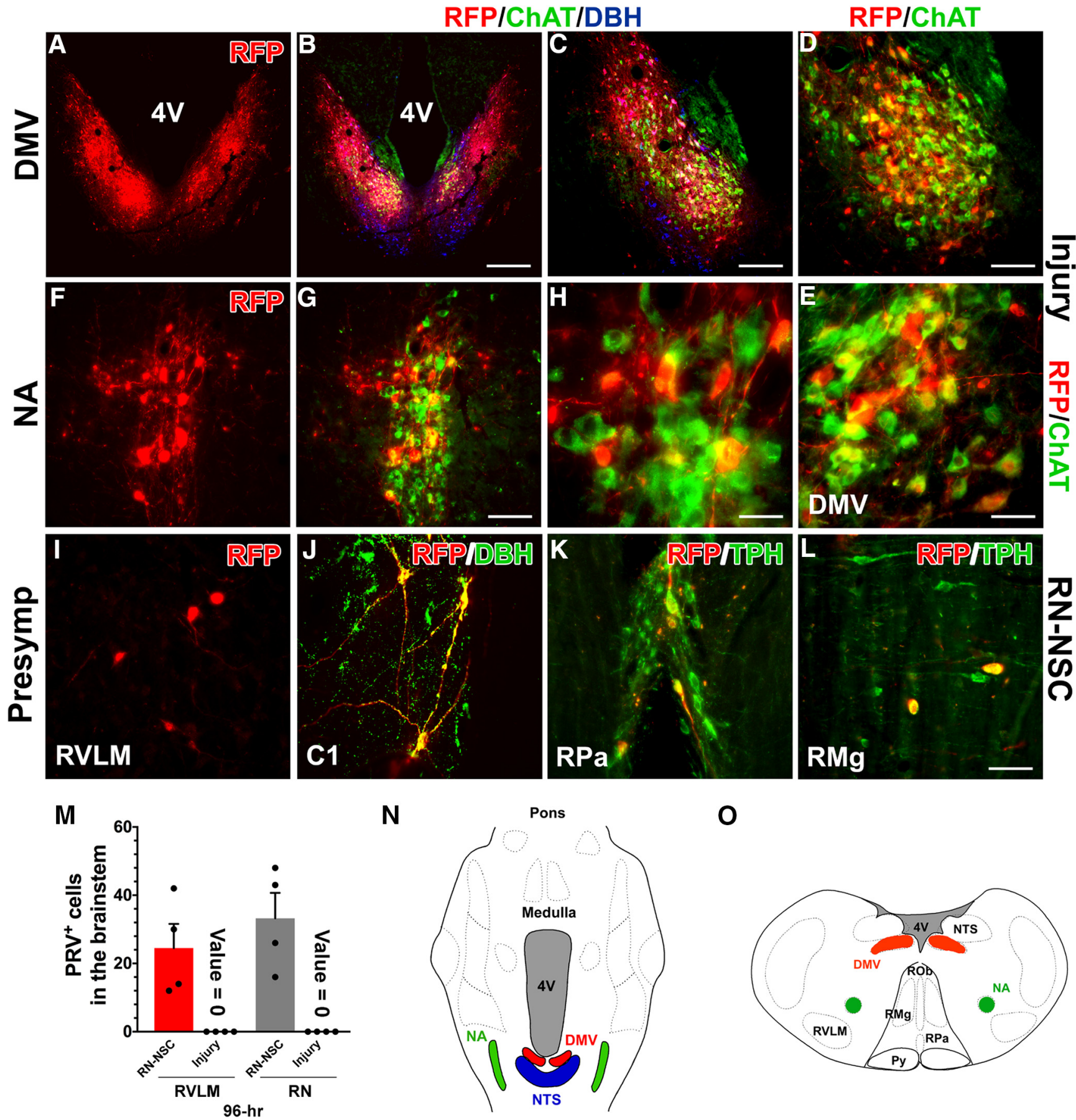

Figure 7. PRV transsynaptic tracing labels neurons in the host brainstem. In either RN-NSC-grafted or injury control animals, PRV-infected neurons were identified within parasympathetic nuclei in the brainstem at both 72 and $96 \mathrm{~h}$ after inoculation ( $n=3$ or 4 per group). $\boldsymbol{A}-\boldsymbol{H}$, Representative photomicrograph shows RFP-immunolabeled PRV-infected neurons $(\boldsymbol{A}-\boldsymbol{E})$, the DMV, and $(\boldsymbol{F}-\boldsymbol{H})$ the NA, which are colabeled with ChAT indicative of cholinergic (green), at $96 \mathrm{~h}$ transportation time. In the DMV, RFP-labeled neurons (red) colocalized with $(\boldsymbol{D}, \boldsymbol{E}) \mathrm{ChAT}^{+}$parasympathetic neurons (green) adjacent to the NTS that contains DBH ${ }^{+}$noradrenergic neurons (blue). $I-L$, However, we observed PRV-infected neurons in sympathetic premotor brainstem regions (e.g., RVLM and caudal RN) only in RN-NSC-grafted rats at $96 \mathrm{~h}$. I, Labeled neurons are detected in the RVLM. J, Infected C1 neurons within this region are DBH ${ }^{+} . \boldsymbol{K}, \boldsymbol{L}$, Infected neurons in the caudal RN, such as the nucleus raphe pallidus ( $\mathrm{RPa}$ ) and raphe magnus $(\mathrm{RMg})$, are colabeled with TPH antibody, indicating serotonergic identity. No infected cell is detected within the presympathetic regions at the brainstem level in animals with injury control. $M$, Quantification of PRV-infected neurons in the RVLM or RN in RN-NSC-grafted or injury control rats at $96 \mathrm{~h}$ after infection. $\boldsymbol{N}, \mathbf{0}$, Parasympathetic and presympathetic structures in the brainstem are outlined in a 2D, (N) horizontal longitudinal or (0) transverse plane; albeit they may be located at different levels. Scale bars: $\boldsymbol{B}, 400 \mu \mathrm{m} ; \boldsymbol{C}, 200 \mu \mathrm{m} ; \mathbf{D}, \mathbf{G}, 100 \mu \mathrm{m}$; $E, 35 \mu \mathrm{m} ; \boldsymbol{H}, 30 \mu \mathrm{m} ; \boldsymbol{L}, 75 \mu \mathrm{m}$.

naturally occurring $\mathrm{AD}$ events during a $24 \mathrm{~h}$ phase (Fig. $9 A, B$ ). Compared with the injury control (number of events: $16.0 \pm$ 4.4), grafting E14 RN-NSCs reduced the incidence of naturally occurring $\mathrm{AD}(4.3 \pm 1.3$; ANOVA $p<0.05$, Fisher's PLSD, $p<$
0.05), whereas grafting SC-NSCs did not $(6.7 \pm 3.7$, Fisher's PLSD, $p=0.12$ ). Rats receiving RN-NSCs did not have a statistically different number of events (Fisher's PLSD, $p=0.91$ ) than those grafted with SC-NSCs (Fig. 9C). In addition, there was no 

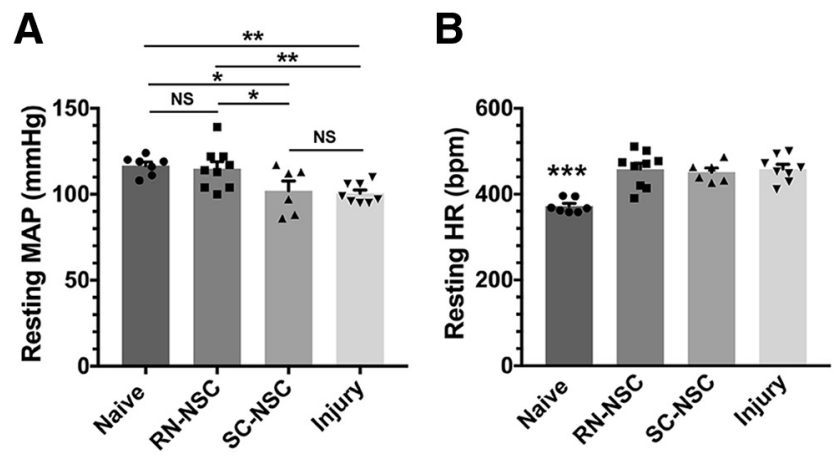

Figure 8. Grafting RN-BSCs improves resting hemodynamic performance following SCI. Telemetric recordings show that, 10 weeks after complete T4 spinal cord transection ( $n=6$ or 8 per group), rats with injury only exhibit significantly $(\boldsymbol{A})$ lower resting MAP (one-way ANOVA, $p=0.005$; Fisher's PLSD: * $p<0.05,{ }^{* *} p<0.01$ ); and $(\boldsymbol{B})$ higher HR (one-way ANOVA, $p=$ 0.001 ; Fisher's PLSD: $\left.{ }^{* *} p<0.001\right)$. However, the resting MAP in rats receiving RN-NSCs is elevated to the level of naive controls ( $p>0.05$ ), while the resting $H R$ is still higher than the normal level ( $\left.{ }^{* * *} p<0.001\right)$. Transplantation of E14SC-NSC $s$ does not affect decreased MAP and increased HR following SCl (both $p>0.05$ vs injury controls; both $p<0.05$ vs naive rats). NS, not significant.

difference in the magnitude of the changes to MAP and HR during the occurrence or the duration of $\mathrm{AD}$ between the three groups (data not shown).

To further examine the severity of $\mathrm{AD}$, we administered $1 \mathrm{~min}$ of CRD, a well-controlled stimulus that mimics constipation, a common trigger of $\mathrm{AD}$ in humans, and that reliably and predictably induces an $\mathrm{AD}$ event experimentally. CRD quickly induced a stereotypical $\mathrm{AD}$ event (i.e., hypertension and bradycardia) in all groups with SCI, regardless of whether they received grafts or not. However, the extent of the MAP increase in response to CRD in animals grafted with either RN-NSCs $(32.6 \pm 3.1 \mathrm{mmHg})$ or SC-NSCs (40.3 $\pm 3.7 \mathrm{mmHg}$ ) was significantly smaller compared with the injury control $(58.5 \pm 5.0 \mathrm{mmHg}$; one-way ANOVA, $p=0.006$; Fisher's PLSD, RN-NSC $p<0.001$, SC-NSC $p<0.01$ ). The change in MAP in rats with RN-NSC grafts was similar to that seen in those with SC-NSCs. There was no difference in the degree of HR decrease during CRD between the three injured groups (Fig. 10A, $C$; one-way ANOVA, $p>0.05$ ). To determine whether cellular grafts influenced the recovery to baseline parameters after the triggering stimulus is removed, we measured the time it took for MAP to return to pre-CRD levels. We found that animals grafted with RN-NSCs exhibited the shortest recovery time $(101 \pm 20 \mathrm{~s})$, whereas rats with injury alone $(168 \pm 7 \mathrm{~s})$ had the longest (one-way ANOVA, $p=0.018$; Fisher's PLSD, $p<$ $0.01)$. The time to recovery to basal MAP levels in animals grafted with SC-NSCs (144 $\pm 16 \mathrm{~s})$ was in between that seen in animals grafted with RN-NSCs and those with an injury but no transplant (Fig. $10 B, D$ ). These results indicate that transplantation of embryonic RN-NSCs diminishes both experimentally induced and naturally occurring $\mathrm{AD}$, whereas grafting SC-NSCs only alleviates the former.

\section{RN-NSCs does not show better effect than BS-NSCs}

To determine whether RN-NSC grafts yielded any functional improvements over those seen with BS-NSCs in our previous study (Hou et al., 2013b), we compared these two datasets for resting hemodynamics and the change of parameters during CRD-induced $\mathrm{AD}$. However, none of them showed significant difference, including resting MAP (RN-NSC $115 \pm 4$, BS-NSC $118.9 \pm 3.7$ mmHg; unpaired $t$ test, $p=0.6$ ) and HR (RN-NSC $458 \pm 15.4$, BS-NSC $418.5 \pm 7.7 \mathrm{bpm}, p=0.09)$, as well as the change in MAP
(RN-NSC 32.6 \pm 3.1 , BS-NSC $38.6 \pm 3.34 \mathrm{mmHg}, p=0.21$ ) and HR (RN-NSC $-120 \pm 26$, BS-NSC $-119 \pm 13.8 \mathrm{bpm}, p=0.97$ ). Because we did not assess naturally occurring AD or the recovery time after CRD-induced AD when we grafted BS-NSCs, we could not compare these indices. Thus, increasing the percentage of 5 -HT neurons in the graft does not appear to produce more extent of cardiovascular recovery other than those yielded by BS-NSCs.

\section{Pharmacological interventions and spinal cord retransection compromise cardiovascular recovery}

To specifically examine the role of 5-HT on the improved cardiovascular function we observed, we assessed the effect of blocking 5-HT receptors signaling using antagonists on hemodynamics. In the spinal cord, 5-HT regulates cardiovascular function via $5-\mathrm{HT}_{2 \mathrm{~A}}$ receptors expressed on autonomic neurons (Doly et al., 2004; Cormier et al., 2010). Inhibiting activation of $5-\mathrm{HT}_{2 \mathrm{~A}}$ receptors using subcutaneous delivery of ketanserin reduced the resting MAP and increased HR in rats grafted with RN-NSCs. Statistical analysis indicated that, compared with the baseline, the resting MAP significantly dropped when three different doses of ketanserin were delivered (basal: $115 \pm 4$, ketanserin $0.5 \mathrm{mg} / \mathrm{kg}$ : $99 \pm 3,1.5 \mathrm{mg} / \mathrm{kg}$ : $98 \pm 2,3.0 \mathrm{mg} / \mathrm{kg}$ : $95 \pm 2 \mathrm{mmHg}$; repeatedmeasures one-way ANOVA followed by post hoc with Bonferroni corrections, all $p<0.01$ compared with the basal); the increased HR was nonsignificant (basal: $458 \pm 14$, ketanserin $0.5 \mathrm{mg} / \mathrm{kg}$ : $489 \pm 14,1.5 \mathrm{mg} / \mathrm{kg}: 486 \pm 8,3.0 \mathrm{mg} / \mathrm{kg}: 469 \pm 9 \mathrm{mmHg})$ Ketanserin administration did not induce significant changes to MAP or HR in SC-NSC-grafted rats and injury controls (Fig. $11 A, B)$. The results demonstrated that RN-NSC, but not SCNSC, grafts provided meaningful serotonergic regulation of recovered resting hemodynamics.

We could not directly assess how inhibiting 5-HT receptors affects CRD-induced AD. After administration of ketanserin, CRD elicited only a subtle pressor response in all three groups of injured animals. This may be due to the necessity of these receptors for sympathetic reflexes or peripheral responses (Danzebrink and Gebhart, 1991; Trueblood et al., 2019). Therefore, we then turned to retransecting the spinal cord below the injury to evaluate the role of RN-NSC grafts in the improved cardiovascular function we observed. This severs axons that extend out of the graft, thus interrupting synaptic connections formed between neurons within the graft and those in the caudal spinal cord. Ten weeks after transplantation of RN-NSCs, cardiovascular parameters were measured, including resting MAP and $\mathrm{HR}$, naturally occurring and CRD-induced AD. Then, the spinal cord was transected 2-3 mm below the graft. One week later, after hemodynamics restabilized, cardiovascular parameters were measured again. We observed that the retransection did not impair the recovered resting MAP (before $108 \pm 1$, after $111 \pm 1 \mathrm{mmHg}$; paired $t$ test, $p>0.05$ ) and HR (before $451 \pm 6$, after $473 \pm 6$ bpm; $p>0.05$ ) (Fig. 11C). Retransection did, however, increase the number of naturally occurring $\mathrm{AD}$ (before $3.8 \pm 1.1$, after $7.2 \pm 1.4, p<0.05$ ) over a $24 \mathrm{~h}$ recording period (Fig. $11 D$ ). Moreover, during CRD stimulation, the changes in MAP and HR were exacerbated (MAP: before $28.3 \pm 6.0$, after $45.6 \pm 7.2$ $\mathrm{mmHg}, p<0.05$; HR: before $-107 \pm 12$, after $-128 \pm 21 \mathrm{bpm}$, $p>0.05$ ) to the level of injury controls (Fig. 11 E,F). This denotes that projections either from or through the RN-NSC graft contribute to alleviating the deleterious sympathetic response. 


\section{A}

Detections MAP plot
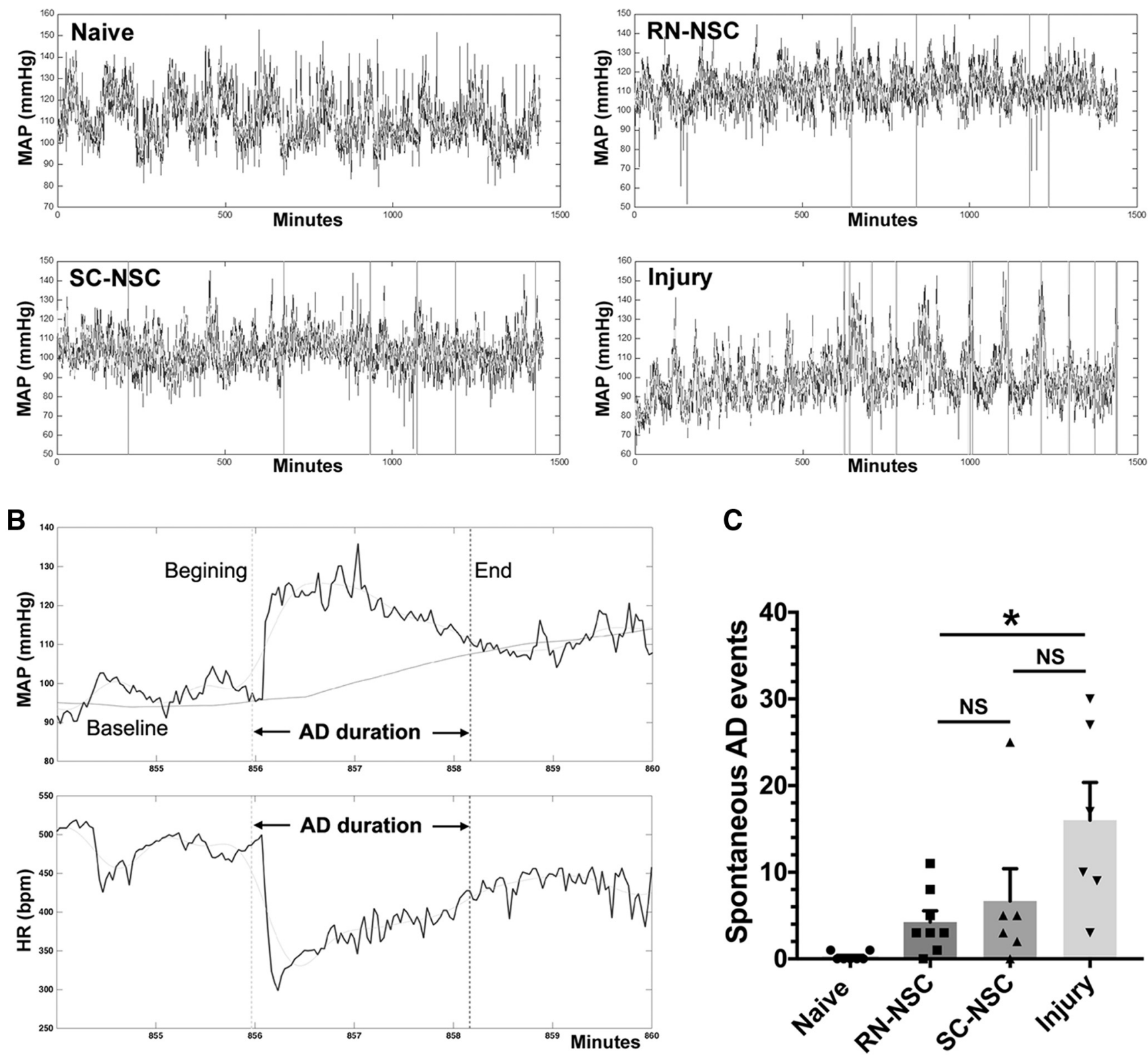

Figure 9. The incidence of naturally occurring AD reduces after grafting embryonic NSC in the injured spinal cord. $A$, Representative MAP tracer is shown over a $24 \mathrm{~h}$ period $(n=6$ or 8 per group). Vertical line indicates an episode of AD, detected by using the algorithm. $\boldsymbol{B}$, A typical spontaneous AD is shown in time-stretched tracers of MAP and HR in a RN-NSC-grafted rat, which is manifested by suddenly increased MAP and simultaneously decreased HR. Vertical dotted lines indicate the beginning and end of AD. Horizontal black line indicates the baseline of MAP. Horizontal gray line indicates a smoothed curve. C, Statistical analysis reveals significantly less events of AD in rats grafted with RN-NSCs than in those with injury alone (one-way ANOVA $p<0.05$, Fisher's PLSD: both $\left.{ }^{*} p<0.05\right)$. Statistically, animals receiving RN-NSCs have no different occurrence of AD compared with those receiving SC-NSC graft ( $\left.p=0.91\right)$. NS, not significant.

\section{Discussion}

Cell transplantation is a promising therapeutic strategy to compensate for the damaged neuronal pathways after SCI and promote functional recovery. In the present study, we implanted $5-\mathrm{HT}^{+}$neuron-enriched RN-NSCs to evaluate the specific effect of rebuilding descending serotonergic regulation on cardiovascular functional recovery. Consequently, grafting RN-NSCs reestablished connections between sympathetic premotor neurons in the brainstem and SPNs in the caudal spinal cord, leading to improved serotonergic control of cardiovascular function.

Central serotonergic modulation of spinal autonomic output neurons is critical for maintaining cardiovascular function. For decades, anatomical and physiological research has disclosed the location of higher centers for hemodynamic modulation (LlewellynSmith, 2009). In particular, neurons in the caudal RN of the brainstem play a specific role in serotonergic regulation of cardiovascular function (Minson et al., 2002). Here, we found that transplanting embryonic RN-NSCs into the lesion of a completely transected spinal cord increased resting blood pressure and attenuated naturally occurring and CRD-induced AD. Although grafting RN-NSCs elevated resting MAP to the normal level, resting HR did not display substantial change. Since parasympathetic pathways regulating the heart are still intact after SCI, which was also denoted by PRV tracing, lack of effect on the $\mathrm{HR}$ in response to improved MAP after grafting is most likely the result of reduced baroreceptor regulation. Previous studies re- 
A
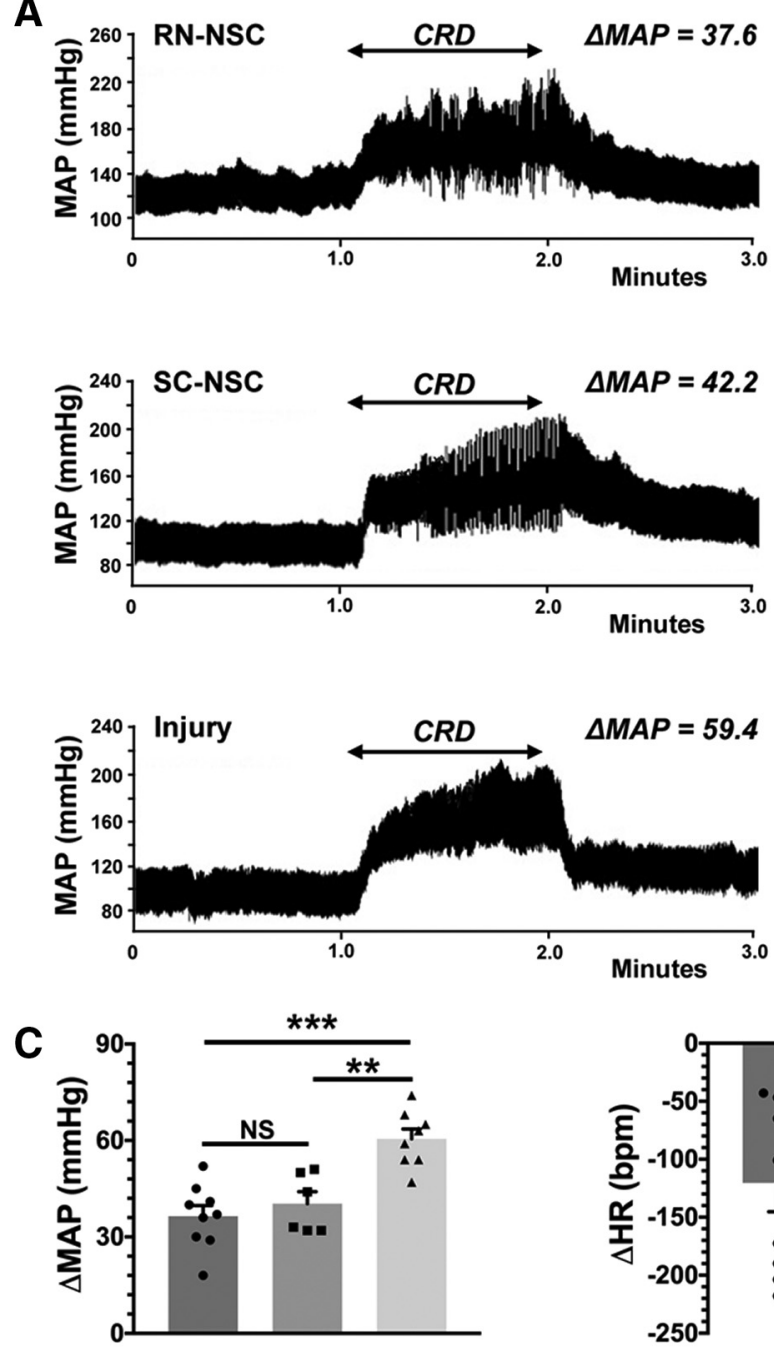

B
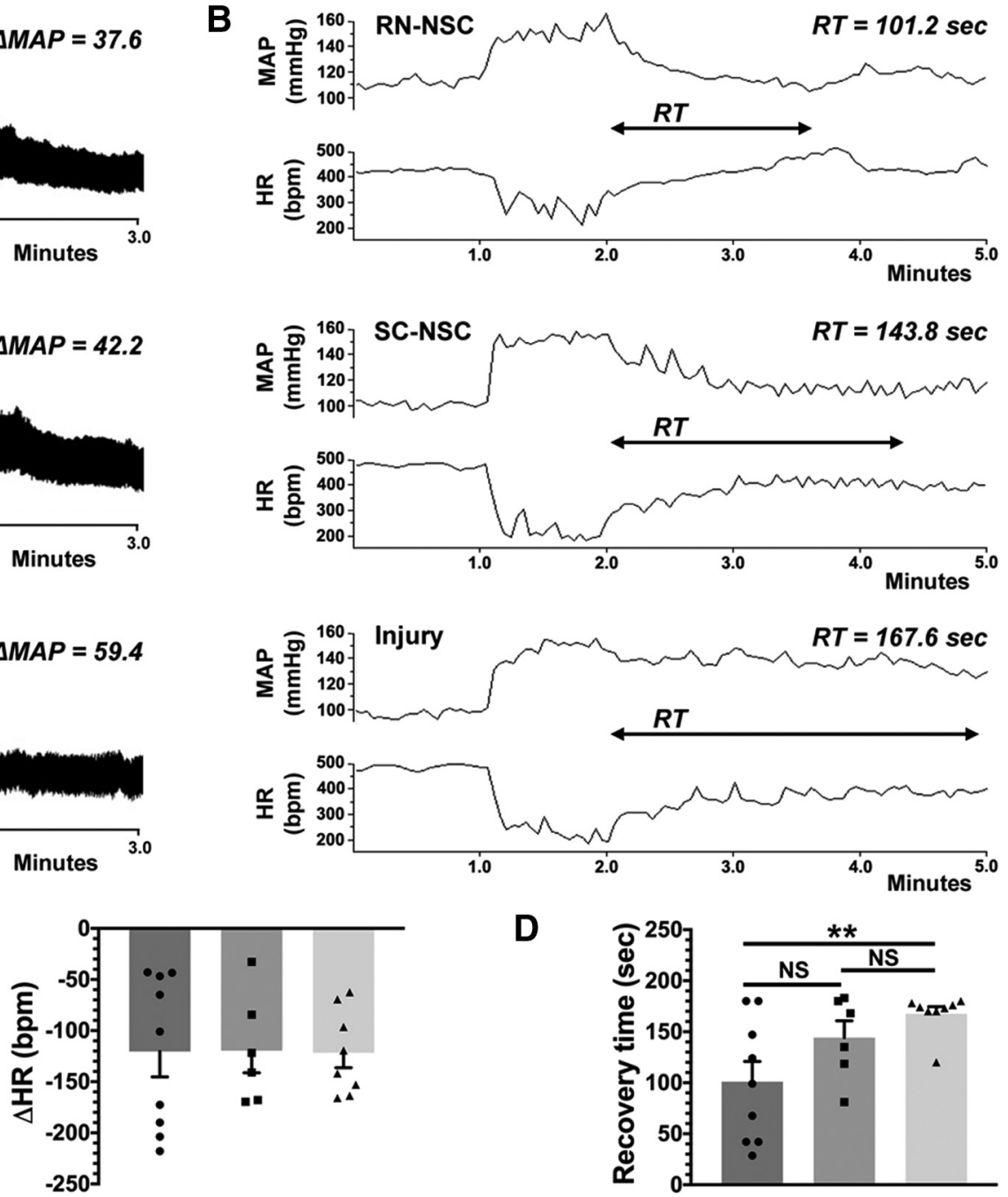

D

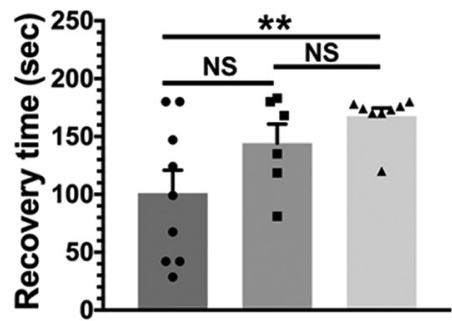

RN-NSC

SC-NSC

Injury

Figure 10. Grafting RN-NSCs mitigates the severity of CRD-induced AD. A,C, An episode of AD develops in response to CRD, which mimics a visceral pain, in all tested rats ( $n=6$ or 8 per group). Compared with animals with injury alone, a lower extent of increased MAP is detected in animals grafted with either RN-NSCs or SC-NSCS (one-way ANOVA, $p=0.006$; Fisher's PLSD, RN-NSCs: ${ }^{* * *} p<0.001$; SC-NSCS: $\left.{ }^{* *} p<0.01\right)$. The change of MAP is nonsignificantly smaller $(p>0.05)$ in RN-NSC-grafted animals than SC-NSC-grafted animals. In contrast, there is no difference in HR change during CRD between NSC-grafted animals and those with injury alone (ANOVA, $p>0.05$ ), although HR decreases in all three groups corresponding to the stimulus. $\boldsymbol{B}, \boldsymbol{D}$, After 1 min CRD duration, the MAP and HR gradually recover to baseline level. Quantitative analysis demonstrates distinct recovery time among the three groups, in which the shortest time is displayed in rats grafted with RN-NSCs and the longest time in rats with injury alone. Statistics indicates significantly shorter recovery time in RN-NSC-grafted rats compared with those with injury alone (ANOVA, $p=0.018$; Fisher's PLSD, ${ }^{* *} p<0.01$ ). However, the recovery time in SC-NSC-grafted rats, longer than RN-NSC-grafted rats but shorter than rats with injury alone, is nonsignificantly different versus both groups (Fisher's PLSD, both $p>0.05$ ). NS, not significant.

ported impaired baroreflex regulation of blood pressure and HR after SCI may be attributed to the compromised sensitivity of arterial baroreceptors (Maiorov et al., 1997; Draghici and Taylor, 2018). Baroreflex sensitivity in people with chronic SCI is approximately half that of able-bodied subjects (Convertino et al., 1991; Koh et al., 1994), which is likely due to the long-term impact of vascular stiffening on baroreceptive arteries (Phillips et al., 2014) and/or long-term generalized deconditioning (van Koppenhagen et al., 2014; van der Scheer et al., 2015). In the present study, the results of increased MAP and unchanged HR suggest that RN-NSC grafts improve sympathetic regulation but have no effect on peripheral vascular pathophysiology and reduced baroreceptor sensitivity. When spinalized rats received SC-NSC grafting, resting hemodynamics were not augmented, consistent with our previous observations (Hou et al., 2013b). This indicates that the implants lack sufficient components that RN-NSCs have to elevate sympathetic vasomotor tone. Previous studies reported that $5-\mathrm{HT}_{1 \mathrm{~A}}$ and $5-\mathrm{HT}_{2 \mathrm{~A}}$ receptors were identified to play a role in regulating blood pressure (Coote, 1990; Lewis and Coote, 1990). In the spinal cord, 5- $\mathrm{HT}_{2 \mathrm{~A}}$ receptor is robustly expressed on SPNs in the autonomic region (Maeshima et al., 1998; Doly et al., 2004). Taking advantage of this, we can manipulate the activity of 5-HT receptors to specifically study how reestablishing serotonergic control of SPNs restores cardiovascular function. Pharmacological blockade of 5- $\mathrm{HT}_{2 \mathrm{~A}}$ receptors with ketanserin eliminated the recovery of resting MAP in rats grafted with RN-NSCs, whereas the intervention did not significantly influence hemodynamic parameters in those either grafted with SC-NSCs or receiving only injury. Thus, these experiments suggest that 5-HT-enriched RNNSC grafts play an important role in control of the basal blood pressure. Furthermore, it precludes the possibility that the effect of drug is mediated via peripheral organs since the cellular and 


\section{Pharmacological interventions of resting hemodynamics}

A

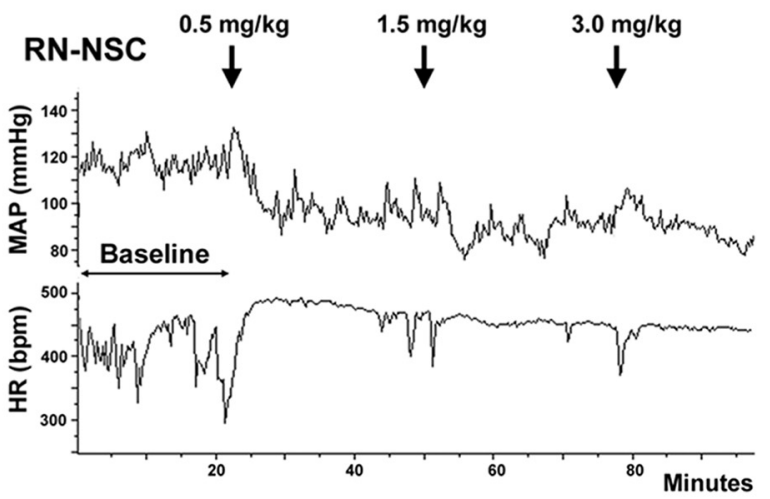

B

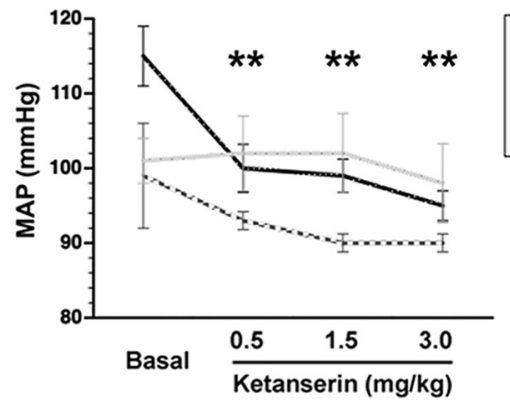

Injury $\quad \downarrow \quad 0.5 \mathrm{mg} / \mathrm{kg} \quad 1.5 \mathrm{mg} / \mathrm{kg} \quad 3.0 \mathrm{mg} / \mathrm{kg}$
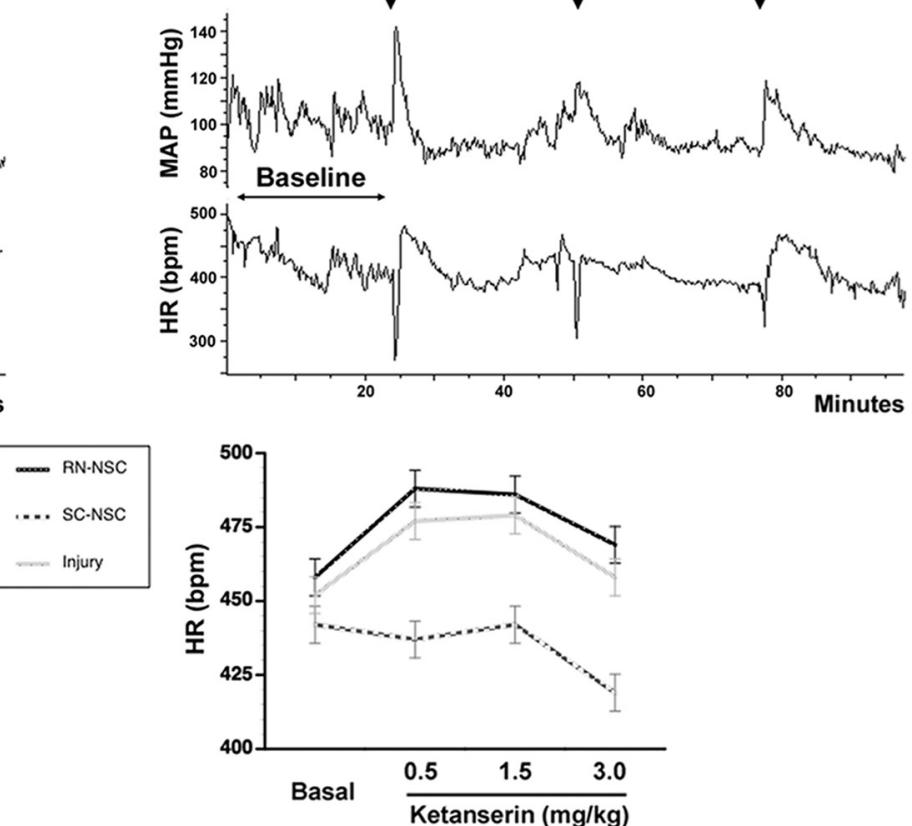

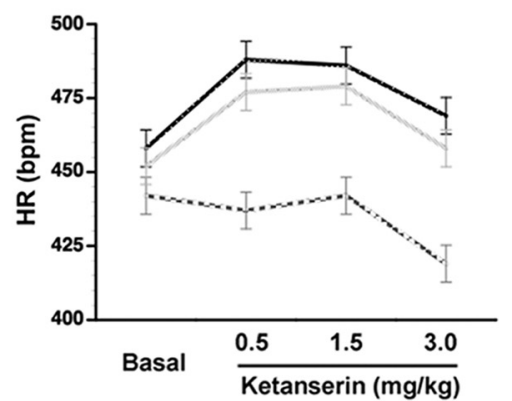

Spinal cord re-transection

C

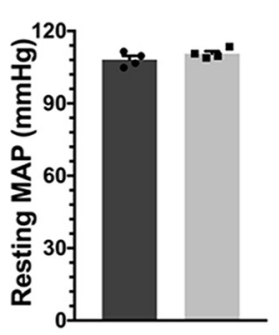

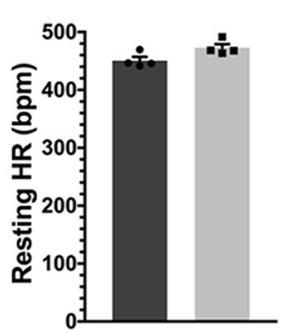

Before Re-Transection
$\mathbf{F}$

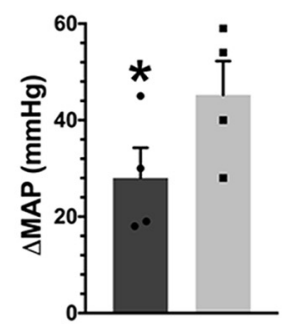

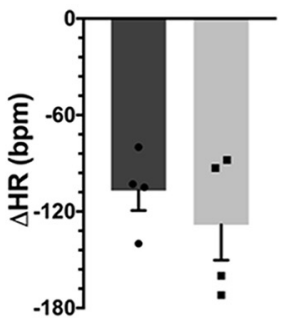

After Re-Transection

E

Before Re-Transection

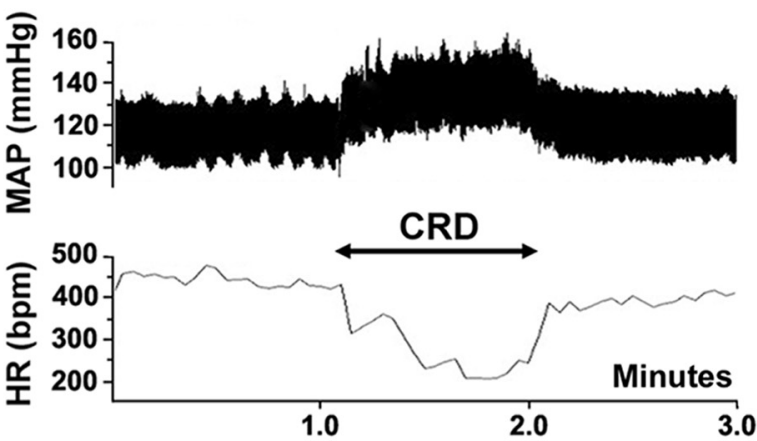

After Re-Transection

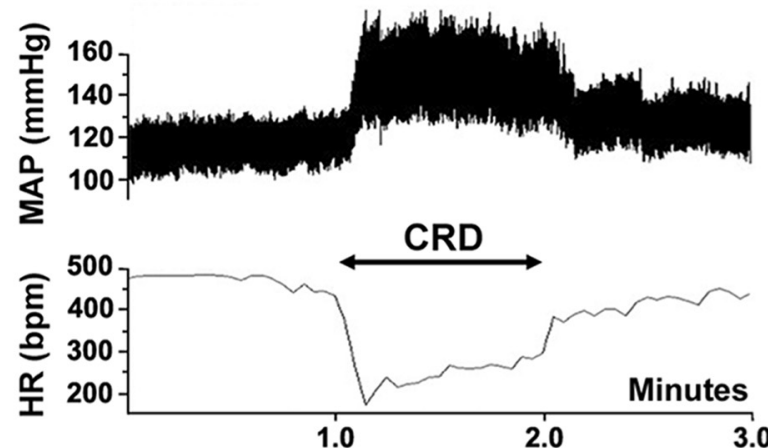

Figure 11. Pharmacological intervention or spinal cord retransection compromises cardiovascular improvements. $A, B$, A serial dose of ketanserin is administered subcutaneously to block 5 - $\mathrm{H}_{2 \mathrm{~A}}$ receptors during hemodynamic recordings ( $n=5$ or 6 per group). $A$, A tracer of continuous recording shows, in response to the drug delivery, the resting MAP reduces in RN-NSC-grafted animals, whereas it does not display obvious change in those either grafted with SC-NSCS or injury controls. B, Statistical analysis confirmed that the resting MAP is significantly decreased (repeated-measures one-way ANOVA with Bonferroni corrections, all ${ }^{* *} p<0.01$ ) and HR is nonsignificantly increased (all $p>0.05$ ) in RN-NSC-grafted rats. In contrast, blocking 5 - $\mathrm{HT}_{2 \mathrm{~A}}$ receptors does not induce meaningful change in either rats grafted with SC-NSC sor injury controls (all $p>0.05)$. $(-\boldsymbol{F}$, After spinal cord retransection below the level of RN-NSC grafts, ( $($ ) the resting MAP and HRdo not change dramatically $($ paired ttest, both $p>0.05)$. D, During $24 \mathrm{hrecordings,} \mathrm{retransection} \mathrm{causes} \mathrm{significantly} \mathrm{more} \mathrm{events} \mathrm{of} \mathrm{naturally} \mathrm{occurring} \mathrm{AD}$ (paired $t$ test, ${ }^{*} p<0.05$ ). $\boldsymbol{E}$, Representative examples of CRD-induced $\mathrm{AD}$ before and after spinal cord retransection $(n=$ 4). A dramatic rise in MAP and decrease in HR can be seen when a balloon-tipped catheter is inflated in the colon. Markedly, the extent of MAP increase and HR decrease is exacerbated after retransection. $\boldsymbol{F}$, Statistical analysis shows that CRD stimulation induces a greater increase in MAP (paired $t$ test, ${ }^{*} p<0.05$ ) and nonsignificant decrease in HR $(p>0.05)$ compared with those before retransection. 
injury controls did not show significant changes in the performance.

Grafting RN-NSCs reduced the frequency of naturally occurring $\mathrm{AD}$ and the severity of $\mathrm{CRD}$-induced $\mathrm{AD}$. Previous experiments showed that, in spinal-intact rats, bulbospinal serotonergic input to SPNs regulates spinal reflexes to keep CRD-induced pressor responses brief and small (Bacon and Smith, 1988; Hasséssian et al., 1993). Here, we saw many $5-\mathrm{HT}^{+}$axons derived from transplanted RN-NSCs topographically projecting to the IML and gray commissure, two main autonomic regions. These serotonergic axon terminals formed synaptic connectivity onto the caudal denervated SPNs. In addition, BDA tracing labeled host brainstem axons regenerating into the graft. The transsynaptic tracing with PRV further supports that $5-\mathrm{HT}^{+}$neurons within the graft formed a novel, compensatory relay circuit to help regain serotonergic control of sympathetic activity. SC-NSCs mitigated the severity of $\mathrm{CRD}$-induced $\mathrm{AD}$, consistent with our previous findings (Hou et al., 2013b). There was a trend of fewer events of naturally occurring $\mathrm{AD}$, albeit no statistical impact versus injury controls. This may be due to variation within the group. It is likely that different compositions of RN-NSCs and SC-NSCs account for distinct neuronal mechanisms following transplantation. Interestingly, when we pharmacologically inhibited $5-\mathrm{HT}_{2 \mathrm{~A}}$ receptors in the spinal cord implanted with RN-NSCs, only very subtle pressor responses were triggered by CRD. This illustrates that this receptor is involved in the spinal sympathetic reflex pathways; inactivation of spinal 5- $\mathrm{HT}_{2 \mathrm{~A}}$ receptor disables cardiovascular response to the noxious visceral stimulation.

Spinal cord retransection to disrupt synaptic connections formed between RN-NSC transplant and caudal neurons diminished recovery of the resting blood pressure and MAP changes in both naturally occurring and CRD-induced AD. However, these values did not reach to the extent to those with injury control, indicating that retransection below the injury/graft only partially eliminated the recovery. One possible explanation for this is that monoamines, such as 5-HT, are known to be involved in both synaptic and volume transmission. There was partial integration of grafts with host tissue, resulting in gaps at the graft-host interface in the majority of animals. While the areas with nice integration would allow for axons to traverse into caudal cord to mediate functional recovery via synaptic transmission, an intriguing question remains: are the 5-HT-mediated effects we observed due to 5-HT secreted from grafted cells and diffusing across areas where there was a gap? Previous investigations found that the effective radius for diffusion of monoamine is $\sim 7-8 \mu \mathrm{m}$ (Fuxe et al., 2010; Borroto-Escuela et al., 2015), which is further than the vast majority of SPNs caudal to the lesion. Additionally, the high expression of collagen and chondroitin sulfate proteoglycans (CSPGs) within the fibrotic and glial scars at the lesion site would limit diffusion of 5-HT. Nonetheless, we cannot directly rule out the possibility that viable cells within the graft produced abundant 5-HT that diffused into CSF (Tadepalli et al., 1977), allowing for longer-distance transport to SPNs. Since recent studies found that stimulating spinal $5-\mathrm{HT}_{2 \mathrm{~A}}$ receptors improves basal hemodynamic and reflexes in SCI rats (Cormier et al., 2010; Trueblood et al., 2019), excess 5-HT in the CNS may maintain a certain degree of cardiovascular performance after serotonergic projections are interrupted.

When early-stage neurons are transplanted into a lesion, they can rebuild injured pathways to relay signal from high levels to the caudal spinal cord. It has been shown that engraftment of embryonic raphe cells (Ribotta et al., 2000; Slawinska et al., 2000) or immortalized serotonergic precursors (Hains et al., 2001) into

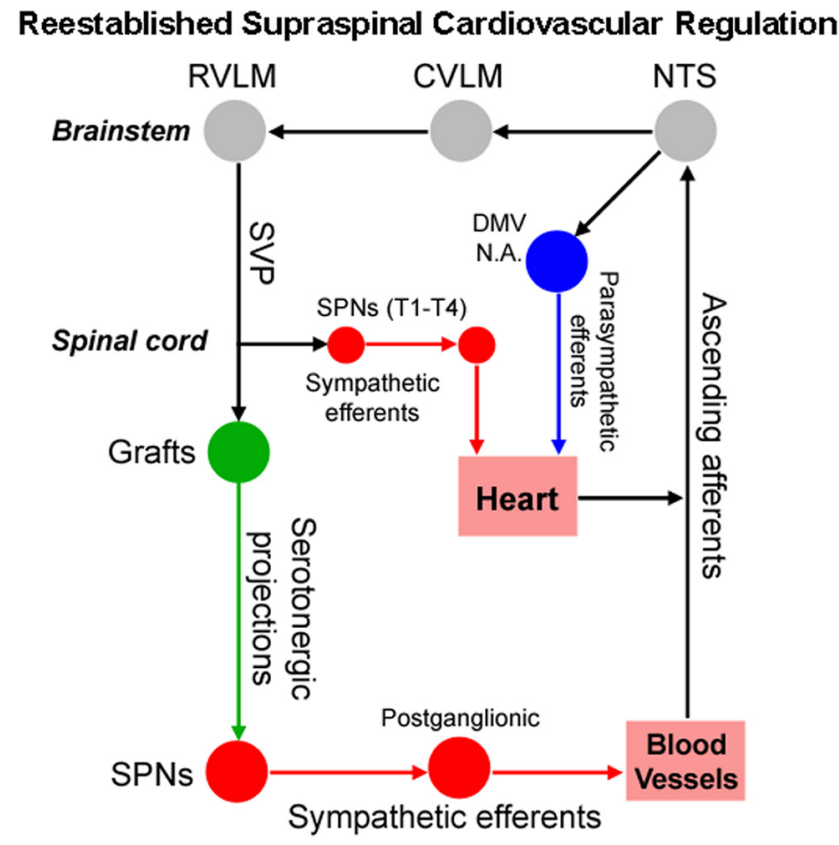

Figure 12. A schematic diagram illustrates the reestablished vasomotor pathways. Primary afferent fibers initiating from arterial chemoreceptors and baroreceptors project excitatory inputs to the NTS in the brainstem. Parasympathetic baroreceptor afferent fibers ascend in the vagus and glossopharyngeal cranial nerves, providing inhibitory inputs to the NTS. As a major recipient of primary sensory cardiovascular information, the NTS precisely regulates hemodynamic performance through the caudal ventrolateral medulla (CVLM) and the RVLM. At the brainstem level, neurons in the RVLM and other presympathetic nuclei originate supraspinal vasomotor fibers projecting to the SPNs in the IML of spinal cord, regulating cardiovascular function. High-level SCl often interrupts this descending control and results in disordered hemodynamics. Transplanting embryonic RN-NSCs into the lesion extends projections onto the SPNs in the caudal spinal cord. On the other hand, the cellular graft facilitates the regeneration of disrupted host supraspinal vasomotor pathways and forms synapses with grafted neurons. Thus, supraspinal autonomic pathways are rebuilt via a relay-based indirect circuit to regulate cardiovascular function following SCI.

the injured spinal cord below the lesion enhances locomotor function, which is attributable to graft-derived new serotonergic innervation of the host spinal cord circuitry (Majczynski et al., 2005). Here, grafting RN-NSCs into the lesion site of the spinal cord appears to successfully reconstruct a relay circuit of serotonergic regulation of sympathetic activity (Fig. 12). This is supported by evidence at multiple levels: (1) grafted early $5-\mathrm{HT}^{+}$ neuron survival and projection to the caudal autonomic regions; (2) synapse formation between new axons and SPNs; (3) regeneration of host supraspinal pathways into the graft; and (4) PRV-labeled 5- $\mathrm{HT}^{+}$neurons in the graft and host brainstem. Furthermore, this is also supported by the abolishment of some recovery with either pharmacological blockade of serotonergic receptors or retransections. Compared with the effect of BSNSCs previously reported (Hou et al., 2013b), RN-NSC grafts did not yield better outcomes in resting hemodynamics and CRDinduced $\mathrm{AD}$. It may illustrate that the small percentage of serotonergic neurons in the BS-NSC grafts was sufficient to mediate recovery.

PRV-infected sympathetic premotor neurons were detected in RN-NSC-grafted rats but not in animals with injury and no graft, indicating that the supraspinal labeling resulted from viral replication and transport via reconnected sympathetic pathways. Interestingly, we observed PRV-infected cholinergic neurons within the DMV and NA, two parasympathetic nuclei in the brainstem, after SCI, regardless of whether a graft was present or 
not. Unlike the gastrointestinal system, which is known to receive both sympathetic and parasympathetic efferents, central autonomic innervation of the kidney and spleen is still controversial. It was postulated that these two organs are controlled by only sympathetic outflow and absent of efferent parasympathetic innervation because early experiments with tract tracing did not find labeled parasympathetic neurons in the brain (Norvell and Anderson, 1983; Schramm et al., 1993; Cano et al., 2001, 2004). In contrast, other studies reported evidence of parasympathetic neuronal fibers in the organs (Korsgren et al., 1993; van Amsterdam et al., 2016). Our data indicate that the kidney receives central control from sympathetic and parasympathetic autonomic divisions. It would be important to further investigate surpaspinal parasympathetic regulation of the kidney.

Previous transplantation studies observed a non-neuronal partition separating the graft in the spinal cord but did not characterize its properties (Sharp et al., 2014). Now we know that the segregation is filled with numerous fibroblasts. As a healing process in response to injury, fibroblasts in the blood vessel, dura, and other connective tissues in the vicinity of lesion site proliferate and migrate toward the lesion, where they secrete a large amount of extracellular matrix proteins, such as collagen, to seal off tissue deficits (Göritz et al., 2011; Fernández-Klett and Priller, 2014; Dias et al., 2018). Immunostaining indicated that fibroblasts may also participate in angiogenesis into the graft while excessive Col-III deposits only in scar area. Accordingly, a major impedance to graft/host fusion may be hypertrophic fibroblastreleased inhibitory factors but not these cells themselves. Despite current progress in cellular grafting, some questions remain that will need further study. How can we attenuate fibrotic scarring to achieve better graft/host integration? Can a certain type of young neurons be more purified to evaluate its specific effects on cardiovascular function? Future research will elucidate these topics for potential clinical applications.

\section{References}

Ahn J, Saltos TM, Tom VJ, Hou S (2018) Transsynaptic tracing to dissect supraspinal serotonergic input regulating the bladder reflex in rats. Neurourol Urodyn 37:2487-2494.

Akhavan M, Hoang TX, Havton LA (2006) Improved detection of fluorogoldlabeled neurons in long-term studies. J Neurosci Methods 152:156-162.

Assinck P, Duncan GJ, Hilton BJ, Plemel JR, Tetzlaff W (2017) Cell transplantation therapy for spinal cord injury. Nat Neurosci 20:637-647.

Bacon SJ, Smith AD (1988) Preganglionic sympathetic neurones innervating the rat adrenal medulla: immunocytochemical evidence of synaptic input from nerve terminals containing substance P, GABA or 5-hydroxytryptamine. J Auton Nerv Syst 24:97-122.

Bonner JF, Connors TM, Silverman WF, Kowalski DP, Lemay MA, Fischer I (2011) Grafted neural progenitors integrate and restore synaptic connectivity across the injured spinal cord. J Neurosci 31:4675-4686.

Bonner JF, Steward O (2015) Repair of spinal cord injury with neuronal relays: from fetal grafts to neural stem cells. Brain Res 1619:115-123.

Borroto-Escuela DO, Agnati LF, Bechter K, Jansson A, Tarakanov AO, Fuxe K (2015) The role of transmitter diffusion and flow versus extracellular vesicles in volume transmission in the brain neural-glial networks. Philos Trans R Soc Lond B Biol Sci 370:20140183.

Calaresu FR, Yardley CP (1988) Medullary basal sympathetic tone. Annu Rev Physiol 50:511-524.

Cameron AA, Smith GM, Randall DC, Brown DR, Rabchevsky AG (2006) Genetic manipulation of intraspinal plasticity after spinal cord injury alters the severity of autonomic dysreflexia. J Neurosci 26:2923-2932.

Cano G, Sved AF, Rinaman L, Rabin BS, Card JP (2001) Characterization of the central nervous system innervation of the rat spleen using viral transneuronal tracing. J Comp Neurol 439:1-18.

Cano G, Card JP, Sved AF (2004) Dual viral transneuronal tracing of central autonomic circuits involved in the innervation of the two kidneys in rat. J Comp Neurol 471:462-481.
Chalmers JP, Pilowsky PM, Minson JB, Kapoor V, Mills E, West MJ (1988) Central serotonergic mechanisms in hypertension. Am J Hypertens 1:79-83.

Charnay Y, Léger L (2010) Brain serotonergic circuitries. Dialogues Clin Neurosci 12:471-487.

Convertino VA, Adams WC, Shea JD, Thompson CA, Hoffler GW (1991) Impairment of carotid-cardiac vagal baroreflex in wheelchair-dependent quadriplegics. Am J Physiol 260:R576-R580.

Coote JH (1990) Bulbospinal serotonergic pathways in the control of blood pressure. J Cardiovasc Pharmacol 15 [Suppl 7]:S35-S41.

Cormier CM, Mukhida K, Walker G, Marsh DR (2010) Development of autonomic dysreflexia after spinal cord injury is associated with a lack of serotonergic axons in the intermediolateral cell column. J Neurotrauma 27:1805-1818.

Cummings BJ, Uchida N, Tamaki SJ, Salazar DL, Hooshmand M, Summers R, Gage FH, Anderson AJ (2005) Human neural stem cells differentiate and promote locomotor recovery in spinal cord-injured mice. Proc Natl Acad Sci U S A 102:14069-14074.

Dampney RA, Tagawa T, Horiuchi J, Potts PD, Fontes M, Polson JW (2000) What drives the tonic activity of presympathetic neurons in the rostral ventrolateral medulla? Clin Exp Pharmacol Physiol 27:1049-1053.

Danzebrink RM, Gebhart GF (1991) Evidence that spinal 5-HT1, 5-HT2 and 5-HT3 receptor subtypes modulate responses to noxious colorectal distension in the rat. Brain Res 538:64-75.

Dias O, Rocha M, Ferreira EC, Rocha I (2018) Reconstructing high-quality large-scale metabolic models with merlin. Methods Mol Biol 1716:1-36.

Doly S, Madeira A, Fischer J, Brisorgueil MJ, Daval G, Bernard R, Vergé D, Conrath M (2004) The 5-HT2A receptor is widely distributed in the rat spinal cord and mainly localized at the plasma membrane of postsynaptic neurons. J Comp Neurol 472:496-511.

Draghici AE, Taylor JA (2018) Baroreflex autonomic control in human spinal cord injury: physiology, measurement, and potential alterations. Auton Neurosci 209:37-42.

Duale H, Hou S, Derbenev AV, Smith BN, Rabchevsky AG (2009) Spinal cord injury reduces the efficacy of pseudorabies virus labeling of sympathetic preganglionic neurons. J Neuropathol Exp Neurol 68:168-178.

Eldahan KC, Rabchevsky AG (2018) Autonomic dysreflexia after spinal cord injury: systemic pathophysiology and methods of management. Auton Neurosci 209:59-70.

Fernández-Klett F, Priller J (2014) The fibrotic scar in neurological disorders. Brain Pathol 24:404-413.

Furlan JC, Fehlings MG, Shannon P, Norenberg MD, Krassioukov AV (2003) Descending vasomotor pathways in humans: correlation between axonal preservation and cardiovascular dysfunction after spinal cord injury. J Neurotrauma 20:1351-1363.

Fuxe K, Dahlström AB, Jonsson G, Marcellino D, Guescini M, Dam M, Manger P, Agnati L (2010) The discovery of central monoamine neurons gave volume transmission to the wired brain. Prog Neurobiol 90:82-100.

Göritz C, Dias DO, Tomilin N, Barbacid M, Shupliakov O, Frisén J (2011) A pericyte origin of spinal cord scar tissue. Science 333:238-242.

Hains BC, Johnson KM, McAdoo DJ, Eaton MJ, Hulsebosch CE (2001) Engraftment of serotonergic precursors enhances locomotor function and attenuates chronic central pain behavior following spinal hemisection injury in the rat. Exp Neurol 171:361-378.

Hasséssian H, Poulat P, Hamel E, Reader TA, Couture R (1993) Spinal cord serotonin receptors in cardiovascular regulation and potentiation of the pressor response to intrathecal substance $\mathrm{P}$ after serotonin depletion. Can J Physiol Pharmacol 71:453-464

Hou S, Duale H, Cameron AA, Abshire SM, Lyttle TS, Rabchevsky AG (2008) Plasticity of lumbosacral propriospinal neurons is associated with the development of autonomic dysreflexia after thoracic spinal cord transection. J Comp Neurol 509:382-399.

Hou S, Lu P, Blesch A (2013a) Characterization of supraspinal vasomotor pathways and autonomic dysreflexia after spinal cord injury in F344 rats. Auton Neurosci 176:54-63.

Hou S, Tom VJ, Graham L, Lu P, Blesch A (2013b) Partial restoration of cardiovascular function by embryonic neural stem cell grafts after complete spinal cord transection. J Neurosci 33:17138-17149.

Hou S, Saltos TM, Iredia IW, Tom VJ (2018) Surgical techniques influence local environment of injured spinal cord and cause various grafted cell survival and integration. J Neurosci Methods 293:144-150.

Jansen AS, Nguyen XV, Karpitskiy V, Mettenleiter TC, Loewy AD (1995) 
Central command neurons of the sympathetic nervous system: basis of the fight-or-flight response. Science 270:644-646.

Kadoya K, Lu P, Nguyen K, Lee-Kubli C, Kumamaru H, Yao L, Knackert J, Poplawski G, Dulin JN, Strobl H, Takashima Y, Biane J, Conner J, Zhang SC, Tuszynski MH (2016) Spinal cord reconstitution with homologous neural grafts enables robust corticospinal regeneration. Nat Med 22: 479-487.

Kalyani AJ, Rao MS (1998) Cell lineage in the developing neural tube. Biochem Cell Biol 76:1051-1068.

Karlsson AK (1999) Autonomic dysreflexia. Spinal Cord 37:383-391.

Koh J, Brown TE, Beightol LA, Ha CY, Eckberg DL (1994) Human autonomic rhythms: vagal cardiac mechanisms in tetraplegic subjects. J Physiol 474:483-495.

Korsgren O, Jansson L, Andersson A, Sundler F (1993) Reinnervation of transplanted pancreatic islets: a comparison among islets implanted into the kidney, spleen, and liver. Transplantation 56:138-143.

Krassioukov AV, Johns DG, Schramm LP (2002) Sensitivity of sympathetically correlated spinal interneurons, renal sympathetic nerve activity, and arterial pressure to somatic and visceral stimuli after chronic spinal injury. J Neurotrauma 19:1521-1529.

Krassioukov AV, Furlan JC, Fehlings MG (2003) Autonomic dysreflexia in acute spinal cord injury: an under-recognized clinical entity. J Neurotrauma 20:707-716.

Krenz NR, Weaver LC (1998) Sprouting of primary afferent fibers after spinal cord transection in the rat. Neuroscience 85:443-458.

Lewis DI, Coote JH (1990) The influence of 5-hydroxytryptamine agonists and antagonists on identified sympathetic preganglionic neurones in the rat, in vivo. Br J Pharmacol 99:667-672.

Llewellyn-Smith IJ (2009) Anatomy of synaptic circuits controlling the activity of sympathetic preganglionic neurons. J Chem Neuroanat 38:231-239.

Lu P, Wang Y, Graham L, McHale K, Gao M, Wu D, Brock J, Blesch A, Rosenzweig ES, Havton LA, Zheng B, Conner JM, Marsala M, Tuszynski MH (2012) Long-distance growth and connectivity of neural stem cells after severe spinal cord injury. Cell 150:1264-1273.

Maeshima T, Ito R, Hamada S, Senzaki K, Hamaguchi-Hamada K, Shutoh F, Okado N (1998) The cellular localization of 5-HT2A receptors in the spinal cord and spinal ganglia of the adult rat. Brain Res 797:118-124.

Maiorov DN, Weaver LC, Krassioukov AV (1997) Relationship between sympathetic activity and arterial pressure in conscious spinal rats. Am J Physiol 272:H625-H631.

Majczynski H, Maleszak K, Cabaj A, Slawinska U (2005) Serotonin-related enhancement of recovery of hind limb motor functions in spinal rats after grafting of embryonic raphe nuclei. J Neurotrauma 22:590-604.

Mathias CJ (2006) Orthostatic hypotension and paroxysmal hypertension in humans with high spinal cord injury. Prog Brain Res 152:231-243.

Minson JB, Arnolda LF, Llewellyn-Smith IJ (2002) Neurochemistry of nerve fibers apposing sympathetic preganglionic neurons activated by sustained hypotension. J Comp Neurol 449:307-318.

Mironets E, Osei-Owusu P, Bracchi-Ricard V, Fischer R, Owens EA, Ricard J, Wu D, Saltos T, Collyer E, Hou S, Bethea JR, Tom VJ (2018) Soluble TNFalpha signaling within the spinal cord contributes to the development of autonomic dysreflexia and ensuing vascular and immune dysfunction after spinal cord injury. J Neurosci 38:4146-4162.

Mironets E, Fischer R, Bracchi-Ricard V, Saltos TM, Truglio TS, O’Reilly ML, Swanson KA, Bethea JR, Tom VJ (2020) Attenuating neurogenic sympathetic hyperreflexia robustly improves antibacterial immunity after chronic spinal cord injury. J Neurosci 40:478-492.

Mouchet P, Manier M, Dietl M, Feuerstein C, Berod A, Arluison M, Denoroy L, Thibault J (1986) Immunohistochemical study of catecholaminergic cell bodies in the rat spinal cord. Brain Res Bull 16:341-353.

Newton BW, Hamill RW (1988) The morphology and distribution of rat serotoninergic intraspinal neurons: an immunohistochemical study. Brain Res Bull 20:349-360.

Norvell JE, Anderson JM (1983) Assessment of possible parasympathetic innervation of the kidney. J Auton Nerv Syst 8:291-294.

Phillips AA, Krassioukov AV, Ainslie PN, Cote AT, Warburton DE (2014) Increased central arterial stiffness explains baroreflex dysfunction in spinal cord injury. J Neurotrauma 31:1122-1128.

Rabchevsky AG (2006) Segmental organization of spinal reflexes mediating autonomic dysreflexia after spinal cord injury. Prog Brain Res 152:265-274.

Rabchevsky AG, Patel SP, Lyttle TS, Eldahan KC, O’Dell CR, Zhang Y, Popovich PG, Kitzman PH, Donohue KD (2012) Effects of gabapentin on muscle spasticity and both induced as well as spontaneous autonomic dysreflexia after complete spinal cord injury. Front Physiol 3:329.

Reier PJ, Stokes BT, Thompson FJ, Anderson DK (1992) Fetal cell grafts into resection and contusion/compression injuries of the rat and cat spinal cord. Exp Neurol 115:177-188.

Ribotta MG, Provencher J, Feraboli-Lohnherr D, Rossignol S, Privat A, Orsal D (2000) Activation of locomotion in adult chronic spinal rats is achieved by transplantation of embryonic raphe cells reinnervating a precise lumbar level. J Neurosci 20:5144-5152.

Schramm LP, Strack AM, Platt KB, Loewy AD (1993) Peripheral and central pathways regulating the kidney: a study using pseudorabies virus. Brain Res 616:251-262.

Sharp KG, Yee KM, Steward O (2014) A re-assessment of long distance growth and connectivity of neural stem cells after severe spinal cord injury. Exp Neurol 257:186-204.

Slawinska U, Majczynski H, Djavadian R (2000) Recovery of hindlimb motor functions after spinal cord transection is enhanced by grafts of the embryonic raphe nuclei. Exp Brain Res 132:27-38.

Soderblom C, Luo X, Blumenthal E, Bray E, Lyapichev K, Ramos J, Krishnan V, Lai-Hsu C, Park KK, Tsoulfas P, Lee JK (2013) Perivascular fibroblasts form the fibrotic scar after contusive spinal cord injury. J Neurosci 33:13882-13887.

Tadepalli AS, Mills E, Schanberg SM (1977) Central depression of carotid baroreceptor pressor response, arterial pressure and heart rate by 5 -hydroxytryptophan: influence of supracollicular areas of the brain. J Pharmacol Exp Ther 202:310-319.

Teasell RW, Arnold JM, Krassioukov A, Delaney GA (2000) Cardiovascular consequences of loss of supraspinal control of the sympathetic nervous system after spinal cord injury. Arch Phys Med Rehabil 81:506-516.

Trueblood CT, Iredia IW, Collyer ES, Tom VJ, Hou S (2019) Development of cardiovascular dysfunction in a rat spinal cord crush model and responses to serotonergic interventions. J Neurotrauma 36:1478-1486.

Tuszynski MH, Steward O (2012) Concepts and methods for the study of axonal regeneration in the CNS. Neuron 74:777-791.

van Amsterdam WA, Blankestijn PJ, Goldschmeding R, Bleys RL (2016) The morphological substrate for renal denervation: nerve distribution patterns and parasympathetic nerves: a post-mortem histological study. Ann Anat 204:71-79.

van der Scheer JW, de Groot S, Tepper M, Gobets D, Veeger DH, van der Woude LH (2015) Wheelchair-specific fitness of inactive people with long-term spinal cord injury. J Rehabil Med 47:330-337.

van Koppenhagen CF, Post M, de Groot S, van Leeuwen C, van Asbeck F, Stolwijk-Swüste J, van der Woude L, Lindeman E (2014) Longitudinal relationship between wheelchair exercise capacity and life satisfaction in patients with spinal cord injury: a cohort study in The Netherlands. J Spinal Cord Med 37:328-337.

Wu D, Klaw MC, Connors T, Kholodilov N, Burke RE, Côté MP, Tom VJ (2017) Combining constitutively active Rheb expression and chondroitinase promotes functional axonal regeneration after cervical spinal cord injury. Mol Ther 25:2715-2726. 